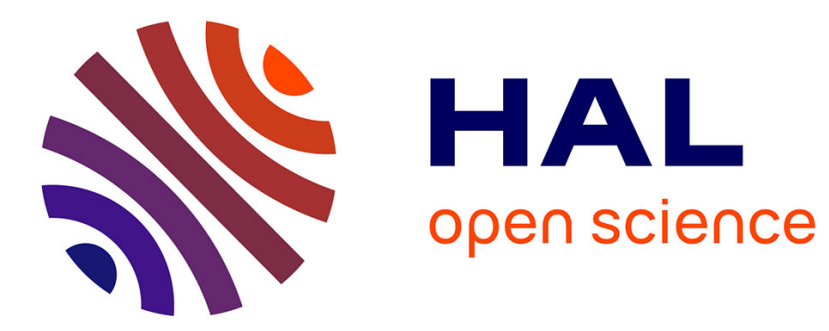

\title{
La stèle d'installation de Śrī Tribhuneśvara
}

Arlo Griffiths

\section{To cite this version:}

Arlo Griffiths. La stèle d'installation de Śrī Tribhuneśvara: Une nouvelle inscription préangkorienne du Musée de Phnom Penh (K. 1214). Journal Asiatique, 2005, 293 (1), pp.11-43. 10.2143/JA.293.1.2002077 . halshs-01910119

\section{HAL Id: halshs-01910119 \\ https://shs.hal.science/halshs-01910119}

Submitted on 1 Nov 2018

HAL is a multi-disciplinary open access archive for the deposit and dissemination of scientific research documents, whether they are published or not. The documents may come from teaching and research institutions in France or abroad, or from public or private research centers.
L'archive ouverte pluridisciplinaire HAL, est destinée au dépôt et à la diffusion de documents scientifiques de niveau recherche, publiés ou non, émanant des établissements d'enseignement et de recherche français ou étrangers, des laboratoires publics ou privés. 


\title{
LA STÈLE D'INSTALLATION DE ŚRĪ TRIBHUVANEŚVARA: UNE NOUVELLE INSCRIPTION PRÉANGKORIENNE DU MUSÉE NATIONAL DE PHNOM PENH (K. 1214)
}

\author{
PAR
}

ARLO GRIFFITHS*

(en collaboration avec J. C. EADE et Gerdi GerschHEIMER)

\begin{abstract}
L’inscription K. 1214 a été découverte le 25 mai 2003 au Duol (tertre) Trabāmnn Samron (dans le village de Bhloen Cheh Radeh Lic, commune de Bhloen Cheh Radeh, district de Țańko, municipalité de Phnom Penh, Cambodge), situé à $25 \mathrm{~km}$ à l'ouest de Phnom Penh, après l'aéroport de
\end{abstract}

* Le présent travail s'inscrit dans le cadre du Corpus des inscriptions khmères (CIK), programme qui vise entre autres à poursuivre l'inventaire des inscriptions du pays khmer établi par George Cœdès et continué par Claude Jacques; sur ce programme, voir BEFEO 90/91 (2003-2004), p. 478-482. Les données sur la découverte de l'inscription sont dues à l'équipe du programme d'inventaire des sites archéologiques du Cambodge (EFEO/ ministère de la Culture et des Beaux-Arts du Cambodge) dirigée par Bruno Bruguier; la description de la pierre, ainsi que les clichés et les estampages utilisés, sont dus à l'Atelier de restauration du musée national de Phnom Penh, dirigé par Bertrand Porte. Gerdi Gerschheimer (qui a aussi effectué une vérification des lectures sur la pierre même en déc. 2004) s'est chargé avec moi de l'édition et de la traduction commentée de la partie sanskrite (avec la note 34), J. C. Eade de l'interprétation des données astronomiques (appendice I). D'autres contributions ponctuelles, dont je tiens à remercier les auteurs, sont signalées en note.

De nombreux collègues ont bien voulu lire tout ou partie de cet article, dans une ou plusieurs de ses versions, et me faire part de leurs observations: Hans Bakker, Christian Bauer, Peter Bisschop, Julia Estève, Michel Ferlus, Philip N. Jenner, Uthaya Veluppillai, Michael Vickery. Je leur exprime ici ma gratitude.

Ce travail n'aurait pu avoir lieu, pour la partie khmère, sans l'aide décisive de Gérard Diffloth (EFEO/Siem Reap), qui a bien voulu m'introduire avec ce texte aux difficultés du vieux khmer, en proposer une glose mot à mot, en expliquer un grand nombre de points. Pour autant, la présente rédaction ne représente pas forcément ses options, et je suis seul responsable, au final, des choix effectués et des erreurs éventuelles.

Ce n'est qu'après la rédaction finale de cet article que j'ai eu connaissance de l'édition de K. 1214 et de sa traduction en khmer par VONG SothEARA (2003). Je remercie Michel Antelme et Olivier de Bernon de m'en avoir commenté quelques points. La réimpression augmentée (déc. 2004) du dictionnaire de Pou (1992) est parvenue trop tard pour que je puisse la consulter. 
Pochentong. Elle a été trouvée au cours de travaux de terrassement, alors qu'une pelleteuse entamait le tertre pour faire une levée le long d'un canal d'irrigation. Le tertre en question, où ont également été trouvés quelques débris de briques, est flanqué, à l'est, d'un bassin ${ }^{1}$.

La pièce a été ramenée le 10 juin 2003 au musée national de Phnom Penh, où, nettoyée par l'Atelier de restauration du musée, elle a été cataloguée sous le numéro $k a$ 3112. Des estampages en ont été réalisés, dont deux sont conservés à l'EFEO (n. 1670 et n. 1671).

\section{Description}

La dalle, haute de $126 \mathrm{~cm}$, large de $35 \mathrm{~cm}$ et d'une épaisseur de $8 \mathrm{~cm}$, est dressée dans une roche gris bleu avec des rehauts ocre qui présente l'aspect d'un schiste.

Sur la face principale, l'inscription, de 31 lignes $(35 \mathrm{~cm} \times 87 \mathrm{~cm})$, est bien conservée, à l'exception de quelques signes emportés par des éclats au bas du côté droit (gauche pour le lecteur). Elle est coiffée par une accolade en très léger relief. En bas, un trait incisé délimite le texte et le pied de la face inscrite porte quelques signes disposés en tous sens. On pourrait penser à un piédroit, mais le motif en accolade évoque plutôt une stèle.

Les quatre premières lignes, en sanskrit, forment une stance de mètre śârdūlavikrīdita (un pāda par ligne), terminée par un fleuron. Les 27 lignes suivantes sont en vieux khmer. Un fleuron de même type que le premier, mais entouré de deux cercles et encadré par des crochets, clôt le texte.

\section{Données historiques et linguistiques}

La strophe en sanskrit relate l'installation d'un Acyuta (Viṣnu), le $28^{\mathrm{e}}$ jour du mois de paușa de l'an śaka 648 , à un moment précisé par la

${ }^{1}$ Dans un second tertre (Duol Lok Yāy), situé à une vingtaine de mètres à l'ouest du premier et flanqué d'un bassin à l'ouest dont la relation au site n'est pas évidente, avait déjà été exhumée une dalle en grès (?) non inscrite, comparable à celle portant K. 1214, ainsi qu'un élément supérieur de piédestal. 
position des sept «planètes» et de l'ascendant. Cette date correspond au mercredi 25 décembre 726 de notre ère (voir l'appendice I).

Il n'y a pas lieu de douter que l'installation en question est celle de la divinité que la partie khmère présente comme l'œuvre pie (punya) de trois personnages, sous le nom de Tribhuvaneśvara. Ce terme évoque certes, à première vue, plutôt un Śiva, mais on verra (n. 34) qu'il peut fort bien s'appliquer également à un Viṣnu. Quant au fait que les trois personnages responsables de l'installation, selon la partie khmère, soient représentés dans la partie sanskrite par le terme yajvan au singulier (yajvanā), il ne semble pas dirimant (voir n. 30).

Le texte khmer consiste essentiellement en un document de donation. Sont mentionnés d'abord, dans une sorte de prologue, les trois fondateurs apparentés avec leurs transactions concernant (principalement) des terrains, sans doute pour le culte du dieu installé (1. 5-8). Sont introduits ensuite trois autres personnages de la génération descendante des trois premiers, qui semblent jouer, avec eux, le rôle de donateurs (9-12). Suivent alors des détails sur divers transferts de terrains (12-14, 14-17, 17 19, 19-21, 21-23), les cédants, les cessionnaires, les prix de cession et les témoins de chaque transaction. Le tout est résumé dans les lignes 23-24. Il semble évident que ces transactions concernent les terrains mentionnés dans le prologue et que ceux-ci font l'objet de la donation par les trois fondateurs (et leurs descendants). Si le texte ne parle explicitement d'une donation que dans le cas d'un Mratāñ Devasvāmi (1. 20), c'est probablement parce que ce dernier ne fait précisément pas partie de ce groupe. Le texte s'achève sur l'autre aspect de la fondation: l'énumération des serviteurs (kñum) acquis (24-31). Cette énumération commence avec les serviteurs féminins $(k u)$, plus exactement avec celles accompagnées de progéniture. Une de ces femmes avait été achetée, et le texte mentionne les détails de cette transaction (cédant, prix, témoins). La liste de serviteurs féminins est complétée par une $k u$ sans enfant. Suivent les serviteurs masculins $(v \bar{a})$ et, pour conclure le document, le total des serviteurs acquis.

Cette inscription a en commun avec l'inscription K. 154 de Phum Komrieng (province de Kandal) un certain nombre de détails qui rendent leur proximité extrêmement probable ${ }^{2}$. Les dates très proches des deux

${ }^{2}$ Elles présentent toutes deux un Mratāñ Devasvāmī et un Poñ Śañk(a)ragaṇa, le topo- 
épigraphes renforcent cette conviction. En effet, K. 154 porte la datation yāte șadbhūtașacchata śakaparigraha navamī roc. jeșța candradivasavāra uttarabhadr. nakșatra nu. CEDÈs (IC II, 124) la traduisait par: «En çaka révolu six cent six, neuvième jour de la lune croissante de Jyeștha, lundi, nakșatra Uttarabhadra(pada)» ${ }^{3}$; et dans la n. 7, il défendait son interprétation de l'expression numérique șadbhūtașacchata comme 606: «et non pas 656 comme le porte mon Inventaire des inscriptions $d u$ Cambodge. Bhüta n'a pas ici le sens d'“éléments"; c'est le verbal bhūta, "réuni à".» L'examen des données astronomiques par BILlARD (à paraître), confirmé par J. C. Eade (voir l'appendice I, p. 34-35), montre qu'il faut revenir à l'interprétation originale de CEDÈs ${ }^{4}$, correspondant à 734 de n. è., soit moins de 8 ans après la date de K. 1214 .

L'inscription présente les traits typiques pour le corpus khmer préangkorien que sont l'utilisation des occlusives sourdes non aspirées précédant une liquide ou une nasale $(k l-, k \tilde{n}-, j l-$, etc.) et l'orthographe des voyelles en syllabes fermées discutée par CEDÈs dans sa Note linguistique (IC II, 2-5) .

Cette épigraphe permet quelques progrès lexicographiques modestes. Elle contient certains mots qui n'étaient pas encore ou très rarement attestés en khmer préangkorien (klai, tor, śada, sahodara, sevabhāra), et elle donne l'occasion de revoir - hélas sans toujours aboutir à une conclusion certaine - l'interprétation de quelques mots difficiles (purușakāra, '(a)val, '(a)dah).

Parmi les données que les historiens du Cambodge ne manqueront pas de vouloir exploiter, figure un complexe de relations de parenté (1. 5-12) dont l'interprétation exacte reste, elle aussi, incertaine.

nyme Hañsapura et le mot '(a)val: ces éléments n'ont pas encore été rencontrés dans d'autres inscriptions préangkoriennes. Voir les notes 47, 49, 56 et 64. La localisation précise du Phum Komrieng dont provient K. 154 n'est hélas pas connue, et il n'a pas été possible de l'indiquer sur la carte ci-jointe (p. 43), où sont marqués les sites d'origine de K. 1214 et de K. 582 (cf. n. 49 et 64).

${ }^{3}$ Donc avec yāte élément participial d'une construction sanskrite (le locatif absolu), dans une datation qui du reste peut être interprétée selon les règles de la syntaxe khmère.

${ }^{4}$ La restitution de la datation plus récente de K. 154 nécessite la révision de quelques suppositions historiques dans l'étude de VICKERY 1998 (par exemple, p. 106, 210 n. 113).

${ }_{5}$ Voir aussi les observations sur les différences entre les états préangkorien et angkorien du vieux khmer dans VICKERY 1998, 85 et suiv. 


\section{Conventions}

L'écart important entre l'état des connaissances du sanskrit, d'une part, du vieux khmer de l'autre, m'a paru justifier une présentation plus élaborée pour ce dernier. Je note dans l'édition du khmer les virāma $(« \cdot \gg)^{6}$ et adopte la convention introduite dans l'épigraphie khmère par JACQUES (1999) pour l'utilisation de «=»; les danda (I) sont maintenus tels quels, qu'ils marquent une ponctuation ou un chiffre; leur interprétation est donnée dans la traduction.

Certains signes utilisés pour les «voyelles indépendantes» dans le système d'écriture indien apparaissent également en khmer comme premier ou deuxième élément d'une ligature - à ma connaissance, c'est toujours le signe $\langle\mathrm{a}-\rangle$ (c.-à-d. la première lettre de l'ordre alphabétique) qui fonctionne comme premier élément de ligature, en deuxième position on peut également avoir d'autres signes de «voyelles indépendantes». De plus, on trouve de telles «voyelles» après des syllabes fermées par le signe d'anusvāra $(\langle\mathrm{m}\rangle)$, où le système indien ne permettrait (en principe) que $\langle\mathrm{mV}\rangle$. Ces phénomènes et les études de phonologie historique montrent que les signes des «voyelles indépendantes» du système indien représentent en fait en khmer une consonne, à savoir l'occlusive glottale, suivie de la voyelle correspondante (voir JАСОВ 1960, 353 et suiv.).

Il n'y a pas à l'heure actuelle d'unanimité sur la translittération de cette consonne $e^{7}$. Je ne connais aucun traitement approfondi de cette matière dans l'épigraphie khmère, mais rappelle les remarques de SHORTO (1971, xii) consacrées au même problème de la translittération dans l'épigraphie mône:

${ }^{6}$ Malgré la redondance de la translittération qui en résulte dans la majorité des cas, il semble important, dans les conditions actuelles, de pouvoir rendre compte du fait qu'une épigraphe comme celle publiée ici présente, par exemple, pour le nom propre Kansec l'orthographe kan.sec (1. 28), n'utilisant pas de ligature $n s$. La notation du virāma, graphème qui peut rester visible alors que sa consonne a disparu, permet de surcroît une translittération plus précise de portions de texte abîmées (cf. 1. 30, début).

7 Cf. entre autres JENNER (1981 et 1982) qui utilise l'apostrophe et le «q», FERLus $(1992,61)$ qui utilise partout le signe phonétique ?, et CEEDÈs (IC) qui, selon le cas, présente la «voyelle» comme telle, la met en exposant, ou y préfixe un trait d'union (ses anak, ${ }^{a}$ nak, sam-ap contiennent tous le même graphème - Pou 1992 emploie une variante du même système, avec un «q qui représente un $a$ suscrit», donc qnak, voir p. XI). 
The vowel support, which is not restricted to syllable-initial position as in Indian languages - in Mon it generally represents a glottal stop-has in most earlier works been transcribed in varying ways according to context, some of them ambiguous. It is here rendered by ' in all positions and not only when final. (Initial vowel symbols, which are allographs of the corresponding sequences of support+vowel, are transcribed in the same way: a regrettable necessity imposed by the inaccessibility of some original texts.) Thus what in Epigraphia Birmanica are transcribed as $\bar{a} r, a b a, p-\bar{a} r$,

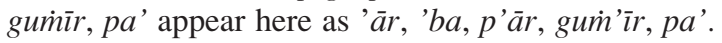

Sur le modèle proposé par SHORTo, je représente ici l'occlusive glottale systématiquement par «'» (p. ex. 'nak, 1. 8), ce qui a pour effet de modifier la physionomie habituelle de nombreux mots (p. ex. 'añ et 'anvaya, 1. 5 et 9). Quant aux possibilités d'allographie décrites par SHORTO, il faut remarquer que je n'ai pas trouvé, jusqu'à présent, d'exemples de l'orthographe dite «support+vowel» au début de syllabe; pour l'occlusive glottale en deuxième position de ligature avec une voyelle autre que le $a$ «inhérent», le corpus vieux khmer montre trois orthographes différentes, que je représente de la façon suivante: (1) «consonne + "initial vowel symbol"» $(k$ 'ol $),(2)$ «consonne + "support" + vocalisation» $(k$ "ol $)$; $(3)$ «consonne + "initial vowel symbol" + vocalisation» $(k, " o l)^{8}$.

Pour l'édition, j'emploie les conventions suivantes:

(...) Entourent les éléments graphiques à identification visuellement incertaine. (a/o) représente ce qui pourrait être lu aussi bien $a$ que $o$.

[...] Entourent les éléments graphiques endommagés ou disparus, restaurés par conjecture.

[[...]] Entourent les éléments graphiques restaurés par conjecture, n'ayant jamais été écrits.

$\langle\ldots\rangle$ Entourent des éléments graphiques annulés par le lapicide.

+...+ Entourent des éléments graphiques ajoutés par le lapicide.

$=$ Réunit des consonnes écrites en ligature mais appartenant à deux mots différents.

virāma.

$\odot \quad$ Fleuron.

${ }^{8}$ Pour les exemples donnés ici, voir la n. 40 ci-dessous. 


\section{Édition}

Sanskrit (l. 1-4)

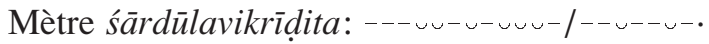

(1) śrīmāñ chrīnidhir ${ }^{9}$ acyutas sugataye kīṭodayo yạ̣ yadā

(2) cāpādau śaśabhrcchatakratugurū mārge rkasaumyārkajāḥ

(3) meșe bhūmisuto ghate bhrgusuto șțāviñśapauṣe tadā

(4) śāke mūrttisamudrakośagaṇite saṃsthāpito yajvanā $\odot$

Vieux khmer (l.5-31)

(5) vrah kamratān. 'añ. śrī tribhuvaneśvara punya mratāñ vinī$\operatorname{ta}(6)$ vin. dan் tāñ sthiradevi ta $\mathrm{p}$ "on. sahodara mratāñ vinītavinna ${ }^{10}$ da(7)n் mratāñ • vinītagaṇa ta klai sahodara mratāñña sanme ni ge (8) pī 'nak=ta sthāpanā vrạ jāhv sre duñ kñum tve daṃrin’ jlan̉ ka'ol· puruṣakāra (9) ge dan' 'anvaya tel ' oy· ta mratāñ cāruvidya dan' mratāñ sucari(10)tānanda dan' mratāñ viditānanda ta kon· mratāñ vinī+ta+gaṇa ${ }^{11}$ kanmoy. sa(11)h(o)dara mratāñ vinītavin. kaṃmoy. ${ }^{12}$ sahodara tāñ sthiradevi $\langle$ gi nā $\rangle$ (12) gi nă ${ }^{13}$ ge 'oy· puruṣakāra ge dan' 'anvaya dan. vrahha $\|^{14}$

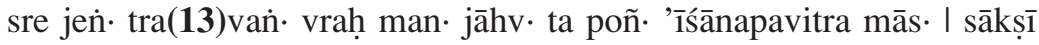

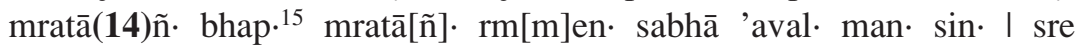
sevabhāra ${ }^{16}$ mana $^{17}$ (15) poñ. śańkrakīrtti (da)n’ poñ. śan̉kragana pañ-

${ }^{9}$ La forme du dhi ressemble beaucoup à celle du vi dans la 1. 3, mais s'en distingue par un petit trait à l'intérieur du signe (voir aussi les dh de vinayavardhana, 1.17 et 19).

10 vinitavinna: variante orthographique de vinitavin. (1. 5-6). Sur ce type de variation $(C \cdot=C C a)$, voir JACOB 1960,360 et 366; JENNER 1981, 2 et suiv.; voir aussi les mots écrits $m$ ratãñ̃na $=$ mratāñ $\cdot(1.7)$ et vrahha $=\operatorname{vrah}(1.12)$.

11 vinī+ta+gana: l'akșara ta est un ajout interlinéaire au-dessus de la ligne, placé correctement entre les akșara nī et $g a$.

12 kammoy: variante orthographique de kanmoy (1. 10).

13 gi nā: l'examen de la pierre semble confirmer que la leçon à la fin de la 1.11 est bien gi nā - et non gi tā ou gi dā -, et que le lapicide, s'étant rendu compte de sa dittographie, l'a biffée.

${ }_{14}$ Il se peut que ce que je lis comme double danda consiste en deux barres verticales barrées.

15 bhap: l'examen de la pierre semble bien confirmer qu'il faut lire ainsi et non gap.

16 sevabhāra: la séquence vabhā pourrait à la limite être lue bhagā. Ma lecture est justifiée ci-dessous, n. 48.

17 mana: le virāma a été oublié, lire man. 


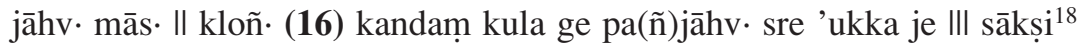
mratāñ kulaśarmma (17) mratāña rmmena ${ }^{19}$ sa(bh)ā 'aval man· sin· ॥ sre man poñ. vinayava[r]dha(18)na ${ }^{20}$ pañjāhv pāda I je III sākṣī poñ. kulabhūṣa po(ñn) bhīmagaṇa (19) mratāñ vivrta I sre man· poñ. vinayavardhana tor poñ $\cdot$ kantān $\cdot(\mathbf{2 0})$ ta mratāñ devasvāmi gi tel· mratāñn pradāna ta vraḥ sākṣi mratā(21)ñn hariśarmma paṃjụ poñ bhīmagaṇa I kloñ tāñ · poñ śarapracanda po(22)ñn nandagup ge sanme ni pañjāhv cpar. ta tāñ sthiradevi sākṣi $\operatorname{mra}(\mathbf{2 3})$ tāñ vivrta poñ. bhīmagana poñ bhadragana poñ nandasena $\|$ sa(24)rvapinnda ${ }^{21}$ gi sre tlon. $10^{22} \mathrm{IIII}$ ton. $40 \mathrm{sl}(\overline{\mathrm{a}}) \operatorname{śada}^{23} \|$

kñum ku sammmrddha kon· ku II ku (25) priy. ${ }^{24}$ kon· ku III ku kandak. kon· ku II ku 'avai rnnnap· kon· ku II ku 'ampān- (26) kon· ku man· duñ. ta

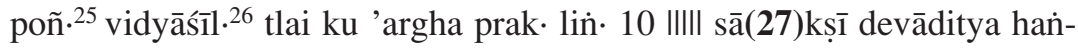

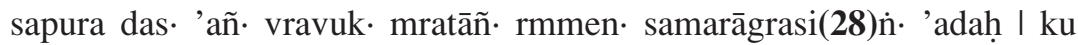
ṇiṣphala kon· ku II ku tamve $\mathrm{e}^{27} \mathrm{ko}[[\mathrm{n} \cdot]] \mathrm{ku} I I I I \mathrm{ku} \mathrm{kan} \cdot \mathrm{sec} \cdot(29) \mathrm{kon} \cdot \mathrm{ku}$ I ku sam’ap· kon· ku III ku thī ko[[n·]] ku II cau IIIII ku pa(30)[n](·hem $)^{28}$ | vā

18 sākși: rien ne permet de supposer qu'on ait voulu écrire un $\bar{l}$ (long) ici, comme dans les 1. 18, 20, 22.

19 mratāña rmmena: la pierre ne présente aucun viräma sur les consonnes finales de ces deux mots, et le point très faible qui surmonte le na a peu de chance d'être une faute pour le virāma attendu. Le lapicide aura oublié ici les virāma qu'il a mis sur les mêmes mots dans les 1.14 et 27.

20 vinayava[r]dhana: la présence d'un akṣara restitué comme étant $r d h a$ semble certaine; voir plus loin, 1. 19.

21 sarvapinda: cf. BHATTACHARYA 1991, 11 n. 58 sur les groupes de consonnes rétroflexes en sanskrit où le deuxième élément est représenté par un signe de l'ordre dental dans l'épigraphie khmère. Voir aussi BARTH 1885, 4 et suiv.

${ }^{22}$ Le chiffre pourrait à la limite être lu gi.

23 śada: ce mot pourrait aussi être lu gada.

${ }^{24}$ priy-: la pierre laisse ouverte la possibilité formelle qu'il faille lire prīy. ou même prīyam.

${ }_{25}$ poñ $\cdot$ : noter la forme abrégée du $\tilde{n}$.

${ }^{26}$ vidyāśsil $l:$ c'est sans doute l'usure de la pierre qui fait hésiter entre un virāma et un anusvāra au-dessus du $l$.

27 tamve: ce mot pourrait aussi être lu kamve. Ma lecture est justifiée ci-dessous, n. 80 .

${ }^{28} \mathrm{pa}[n](\cdot h e m)$ : la syllabe lue hem pourrait aussi être $n \cdot ;$ du viräma de la consonne qui doit précéder, on ne distingue que l'extrémité droite, qu'on pourrait prendre aussi pour un anusvāra. La conjecture ici adoptée (et justifiée ci-dessous, n. 83) nécessite un développement sur la mise en page de l'épigraphe en relation avec l'aspect physique de la pierre. La largeur de la surface écrite visible diminue à partir de la 1.27 sur le côté droit (pour le 
mukhamātra | vā slac· I vā caṃho I vā ṇiṣthura I vā nanda(31)[bha]kti | sarvapiṇda gi kñum phon் 40\|\|$\| \odot$

\section{Signes en bas de la stèle}

Simples exercices de plume (?), dépourvus de contexte et disposés en tous sens, les signes figurant en bas de la stèle ne sont pour la plupart pas interprétables de façon certaine. On distingue clairement, tout en bas, yasya suivi de deux signes; peut-être, perpendiculaire à cet essai, natī; sans doute un graphème ṇa inversé (en haut à droite).

\section{Traduction annotée}

(1-4) Alors que se lève, présentement, le Scorpion (kịta-udaya), que la Lune (śaśabhrt) et Jupiter (śatakratu-guru, le guru d'Indra) sont au début du Sagittaire (cāpa), le Soleil (arka), Mercure (saumya) et Saturne (arkaja, fils du Soleil) dans le Capricorne (mārga). Mars (bhümi-suta, fils de la Terre) dans le Bélier (meșa), Vénus (bhrgu-suta, fils de Bhrgu) dans le Verseau (ghaț), en pauṣa vingt-huitième, en (l'an) śaka compté par les (8) mūrti, les (4) océans et les (6) enveloppes ${ }^{29}$, le patron (yajvan) ${ }^{30}$ a

lecteur), et à partir de la 1.28 à gauche. La pierre y présente deux importantes épaufrures dont on peut montrer qu'elles sont antérieures à la gravure. La séquence samarāgrasiñ ne laisse aucun doute (voir ci-dessous, n. 94) sur l'absence d'akșara à droite de si (fin de la 1. 27), et à gauche de $\dot{n}$. (début de la 1.28 , où l'absence de signe est de toutes façons patente). De même, la séquence $\mathrm{ku} \mathrm{kan} \cdot \mathrm{sec} \cdot \mathrm{kon} \cdot \mathrm{ku}$, complète telle quelle et visiblement sans akșara disparus à gauche de kon, ne donne aucune raison de supposer une lacune à droite de kan.sec. Il s'ensuit que le lapicide a suivi l'aspect de la pierre et qu'il n'y a pas à postuler la perte d'un ou deux akșara à droite de $p a$ (1. 29). Cependant, un décollement secondaire de la pierre à gauche est responsable de la disparition d'un akșara au début de la 1.30 (pour lequel je conjecture $n \cdot$ ) et du haut d'un aksara (bha) au début de la 1. 31 . Ces constatations ne laissent hélas aucune chance de profiter d'une lacune suffisante pour résoudre le problème d'interprétation discuté dans la n. 68 ci-dessous.

${ }^{29}$ Sur la date en question et les données astronomiques, voir l'appendice I. Le millésime (648) est exprimé, comme d'habitude, à l'aide d'un chronogramme. Y figure le terme kośa dans le sens «six», sur lequel nous renvoyons à BHATTACHARYA 1991, 45 [117] et envisageons de revenir. Les mots signifiant «océan» ont en général la valeur «4» (la valeur «7», également possible a priori, est ici exclue par la configuration planétaire). Sur mūrti «8», voir BERGAIGNE 1882, 154.

${ }^{30}$ Il paraît hors de doute que la stance sanskrite parle de la même installation (cf. saṃsthāpita, 1. 4) que celle qui est évoquée au début de la partie khmère (cf. sthāpanā, 1. 8). Le singulier de yajvana, alors que le khmer semble insister sur le fait que l'installation de 
installé, en vue d'une condition heureuse, le fortuné Acyuta ${ }^{31}$, réceptacle de la Fortune ${ }^{32}$.

(5-7) Mon haut Seigneur le sacré ${ }^{33}$ Śrī Tribhuvaneśvara ${ }^{34}$ [est] l'œuvre pie du Mratāñ Vinītavin avec la Tāñ Sthiradevi, cadette uté-

la divinité a été effectuée par «trois personnes à parts égales» (sanme ni ge pī 'nak), pourrait certes faire obstacle à cette identification. Mais diverses solutions permettent de lever cette difficulté, parmi lesquelles la suivante (suggérée par Peter Bisschop) nous paraît la plus plausible: yajvanā s'appliquerait distributivement à chacune des trois personnes, qui s'identifierait ainsi comme "patron». Serait ainsi suggéré, du reste, que les bénéfices de l'acte méritoire en question seront bien récoltés par chacune de ces trois personnes.

31 Appelé Acyuta dans cette stance, la divinité dont la stèle célèbre l'installation est très certainement un Viṣnu. Outre que c'est là le référent le plus courant de acyuta en sanskrit, l'association répétée avec le terme śri au début du premier pāda semble bien faire allusion à la parèdre Śrī de Viṣnu. Du reste, le terme acyuta apparaît encore dans au moins deux autres inscriptions préangkoriennes comme désignation d'un Viṣnu: dans la stèle K. 22 ( $1^{\text {re }}$ moitié du $_{\text {VII }}{ }^{\mathrm{e}}$ s.), il renvoie à la partie vishnouite d'un Harihara (st. I: harācyutau ... pārvatīśrīpatitvena; st. IV: śañkarācyuta); l'inscription K. 447 (657 de n. è.), d'obédience clairement vishnouite (pāñcarätra), relate l'installation de bhagavant acyuta (st. X). Il est probable que c'est également le Viṣnu appelé Campeśvara que désigne acyute de la 1.5 de l'inscription K. 428, de 761 de n. è. Dans le corpus angkorien, on rencontre acyuta en référence à Viṣnu dans les inscriptions K. 675 du Xe s., st. XI (avec jeu de mots); K. 172, du XI ${ }^{\mathrm{e}}$ s., st. V (avec sans doute un double sens); K. 260N, du XI ${ }^{\mathrm{e}}$ s. śaka, st. I. Voir aussi la n. 34.

32 Deux points de la séquence sugataye kītodayo yam nécessitent un commentaire. Le composé kịtodayah, «lever du Scorpion», indique le moment de l'événement par la donnée du signe du zodiaque apparaissant à l'horizon oriental, c'est-à-dire de l'ascendant (lagna; voir ci-dessous, p. 31). Il est assez inhabituel de voir cette donnée accompagnée par un déictique (ayam), qu'on construit plus volontiers avec la divinité, destinée, elle, à rester en compagnie de l'inscription; mais l'enchâssement de ce déictique dans la relative (yadā ...) incite à le rapporter néanmoins au lever du Scorpion. Quant à sugataye («en vue d'une condition heureuse»), il se construit naturellement avec samsthāpita de la principale; mais il n'est pas exclu qu'il faille le rapporter également, appliquant le dehalī-dīpanyāya, «maxime de la lampe sur le seuil» (éclairant à la fois l'intérieur et l'extérieur), au lever du Scorpion, souligné ainsi comme moment favorable (lagna!).

Pour résumer, la stance ne laisse pas de présenter quelques difficultés (cf. aussi supra n. 30), la principale étant le placement erroné de Jupiter (voir ci-dessous p. 34), et on ne saurait tout à fait exclure que l'auteur en soit plus versificateur que poète.

33 Sur les difficultés de traduction que pose le titre «V. K. A.», voir JaCQUes 1986, 317 et suiv.; sur le titre même, qui peut aussi dénoter des rois, mais qui doit ici dénoter la divinité, voir VICKERY 1998, 143, 177 et suiv.

34 Alors que Acyuta, dans la stance sanskrite, désigne la divinité installée de façon générale, en permettant de savoir qu'il s'agit d'un Viṣnu, Tribhuvaneśvara du khmer est très probablement son «nom propre». Il est vrai que ce terme, «le Seigneur des trois mondes», n'a pas encore été relevé dans le corpus des inscriptions du pays khmer comme un nom de Viṣnu. Le seul Tribhuvaneśvara/Tribhuvaneśa d'époque préangkorienne relevé

Journal Asiatique 293.1 (2005): 11-43 
rine $^{35}$ du Mratāñ Vinītavin, [et] avec Mratāñ Vinītagaṇa, beau-frère uté$\operatorname{rin}^{36} \mathrm{du}[$ dit] Mratāñ.

dans les index disponibles est celui de K. 359 ( $\mathrm{VI}^{\mathrm{e}}$ siècle, sanskrit); BARTH [ISC 18-31] n'en précise pas la nature, mais une nouvelle traduction des stances II-III de cette inscription (à paraître ultérieurement) suggère fortement qu'il s'agit d'un Śiva. Des Tribhuvaneśvara mentionnés à l'époque angkorienne, aucun, semble-t-il, ne peut être qualifié de vishnouite: sont assurément ou très probablement çivaïtes les Tribhuvaneśvara de K. 184 (921 de n. è.) [BEFEO 31, 12 = CEEDÈs 1989, 14 et suiv.] et de K. 235 (1053 de n. è.) st. LV [BEFEO 43 (1943/46), 56 = CEEDEs 1992, 167 et suiv.], comme aussi le Śrī Tribhuvaneśvaradeva de K. 449 (1069 de n. è.); rien ne peut être affirmé avec certitude des $k a$ mratén jagat śrí tribhuvaneśvara de K. 418A (1166/67 de n. è.) ou de K. 293.22, 1.4 (XII s. śaka). Quant au fameux Tribhuvanamaheśvara de Banteay Srei, installé en 967 de n. è. (voir st. XLIV de la stèle K. 842, et 1.19 et 20; voir aussi K. 570 st. XI, 1. 24-25, 28, 3334 , 40-41 et 43-44; K. 619-620, st. XXVIII) et dont le culte semble encore connu sous son nom d'origine en 1306 (cf. K. 569 [partie khmère de l'inscription K. 568], 1. 11-12), c'est la composante maheśvara qui en marque le caractère çivaïte, indéniable.

La seule terminaison en ${ }^{\circ} i$ śvara, en effet, pour être plus souvent accolée à un nom de Śiva, ne lui est pas réservée, pas plus au Cambodge qu'ailleurs (de même, l'épithète bhagavant n'est pas confinée à la sphère vishnouite: cf., entre autres, les inscriptions de Citrasena du type K. 122, qui mentionnent sa dévotion pour bhagavataś śambhoh). Nous avons vu ci-dessus (n. 31) qu'on connaît au moins un Viṣnu préangkorien avec un nom ainsi formé: Campeśvara. D'autres cas sont possibles (cf. VICKERY 1998, 142-143, hélas pas toujours convaincant). Quant à la maîtrise des «trois mondes» (tribhuvana, trailokya), elle appartient, selon l'obédience du dévot, à l'une ou l'autre de ces divinités: un Viṣnu destructeur de Madhu fut installé en 921 dans la tour centrale de Pr. Kravan, sous le nom de Trailokyanātha (cf. K. 270 S, 1. 5 [IC IV, 69]), et rien ne s'oppose à ce que le V. K. A. Śrī Tribhuvanasvāmi installé le même jour dans une autre tour de ce complexe (K. 269, 1. 1-4 [IC IV , 74]) soit lui aussi un Viṣnu; de même, la divinité dont l'inscription K. 291 relate l'installation, en 910 de n. è., sous le nom de Śrī Trailokyanātha, est bien un Viṣnu (cf. st. X, bhagavān ... mādhavah); enfin, on notera, parmi les nombreux théonymes dont le premier membre signifie «les trois mondes», un K. J. Tribhuvanasaugateśvara et un K. J. Tribhuvanavaiṣnaveśvara (K. $284 \mathrm{~N}^{\circ} 8$ et 10). La maîtrise des trois mondes peut à juste titre être rapportée à Viṣnu, qui les a conquis par ses trois enjambées: cf. K. 35 ( $\mathrm{X}^{\mathrm{e}} \mathrm{s}$.), st. II, où le théonyme Śrīlokanātha de Hari est justifié par le fait que sa gloire est répandue dans les trois mondes (tribhuvanapravikïnṇakirttih) et mis en rapport avec ses trois pas, comme déjà dans la st. I.

Enfin et surtout, le terme Tribhuvaneśvara, en Inde même, s'il désigne sans doute le plus souvent Śiva, peut également référer à Viṣnu, comme l'attestent les passages suivants de la littérature épique ou puranique, que Peter Bisschop nous a obligeamment communiqués: Mahābhārata, passage *1401 inséré dans 3 mss après 7.170.40, 1. 9; Lingapurāṇa 1.74.19d, 2.6.82b; Vāmanapurāṇa 51.16; Vāyupurāṇa 23.95b. En résumé, rien ne s'oppose au caractère vishnouite de la divinité dont K. 1214 relate l'installation, et dont le nom serait Tribhuvaneśvara.

${ }^{35}$ L'expression sanskrite sahodara «du même ventre» (d'où, plus généralement, «utérin» [?]) est assez rare dans l'épigraphie khmère. Elle n'était pas attestée dans le corpus préangkorien jusqu'ici, bien que le sahutra de K. 78, 1.8 [IC VI, 12], que JeNNER 
1981, 317 ne savait pas expliquer (rien chez Pou 1992 et LONG SEAM), semble en être une khmérification. On rencontre sahodara (en contexte khmer) dans l'inscription angkorienne K. 235, D 1. 25 [BEFEO 43, 89 = CEDÈs 1992, 200], et la même combinaison avec p'on se trouve en forme angkorienne dans K. 91, B 1. 29 [IC II, 130] phaqvan sahodara (CCEDÈs, p. 133: «frère cadet utérin»); on peut comparer aussi ekodarī, K. 720, b 1. 5 [IC V, 212] (CEDÈs, p. 214: "parents utérins»). Les deux termes sahodara et ekodarī sont peut-être des équivalents du mot vieux khmer kaṃton (voir n. 42).

${ }^{36}$ Le mot klai ne semble pas avoir été relevé auparavant dans les inscriptions préangkoriennes. Il n'est pas donné par LONG SEAM; POU 1992, 129 ne le connaît que sous sa forme angkorienne khlai («spouse's brother or sister»), attestée dans K. 956, 1.18 [IC VII, 130] (khlai) et K. 521S, 1. 10 [IC IV, 168] (khlaiy).

Dans une de ses «Notes sur Tcheou Ta-Kouan», CEEDÈs $(1918,8=1989,60)$ discute le renseignement du voyageur chinois (qui visita le Cambodge en 1296-97) selon lequel «oncle maternel se dit tch'e-lai; mari de la tante paternelle, également tch'e-lai». Selon CEDÈs, tch'e-lai «est vraisemblablement une transcription du mot khlai qui revient à plusieurs reprises dans les inscriptions d'Ańkor Vat et désigne effectivement un lien de parenté». Pelliot $(1951,69)$ a approuvé l'idée de voir dans tch'e-lai le khlai du moyen khmer. Les attestations de cette époque sont IMA 37, 1. 17 et 39, 1. 52: LEWITZ (1974, 313 et 324) traduit "parents par alliance» et «belle-sœur», là où AYMONIER (1904, 309 et 311) avait traduit «beau-fils» et «bru». CEDÈs doutait des traductions de ce dernier, «le terme (kón) presà employé actuellement dans le sens de beau-fils (ou belle-fille) étant attesté dans l'épigraphie dès le début du XI ${ }^{\mathrm{e}}$ siècle». Il ajoutait: «Je crois plutôt que khlai est une forme ancienne ou simplement équivalente de thlai, qui signifie actuellement "beau-frère" ou "belle-sœur"». Il concluait en remarquant «que 1" "oncle maternel" et le "mari de la tante paternelle", auxquels s'appliquait selon Tcheou Ta-kouan la désignation de tch'e-lai, sont tous deux les "beaux-frères" du père. Si le Chinois ne s'est pas embrouillé dans tous ces termes de parenté, il faut supposer que depuis son époque le mot khlai a subi une évolution sémantique». Voir aussi YANG Baoyun 1994, 230.

Que klai/khlai soit une forme ancienne ou équivalente de thlai (cf. HEAdLEY et al. 1977, 360 /tlay/ «in-law») paraît certain, mais je ne suis pas sûr qu'il s'agisse d'une «confusion entre occlusive gutturale et occlusive dentale devant semi-voyelle» (CEDÈs, ibid., n. 2; cf. VICKERY 1998, 249 pour les exemples tñam $=$ kñum et $t m e r=k m e r$ ), d'une dissimilation (comme le propose LewITz 1974, 313 n. 7), ou plutôt d'un cas de contamination du terme de parenté par celui pour «cher, de haute valeur», résultant dans l'homonymie du mot /tlay/ dans la langue moderne. La dernière interprétation est celle de Gérard Diffloth, qui m'a obligeamment informé que klai est effectivement la forme héritée du terme de parenté, comme le montre la linguistique comparée.

Je n'arrive à expliquer le complexe de relations où figure klai sahodara qu'en admettant que klai désigne bien le beau-frère. Ceci étant, parmi les situations imaginables, la suivante me paraît la plus plausible: la Tāñ Sthiradevi et le Mratāñ Vinītavin sont nés du même père et de la même mère. Cette mère a eu, avec un autre homme, un fils, Mratāñ Vinītagaṇa, frère utérin (mais pas consanguin) de la Tāñ Sthiradevi et du Mratāñ Vinītavin. Il aura épousé une sœur (qui n'apparaît pas dans l'inscription) du Mratāñ Vinītavin et de la Tāñ Sthiradevi, devenant ainsi leur «beau-frère utérin». Quant à ses enfants, ils sont bien les «neveux utérins» (1. 10 et suiv.) de la Tãñ Sthiradevi et du Mratāñ Vinītavin, voir n. 42 ci-dessous. On aurait donc là un exemple de mariage endogamique avec la demi-sœur utérine (ou, éventuellement, la cousine parallèle matrilatérale). Notons que la 
(7-12) Ce sont eux trois ${ }^{37}$ qui, à parts égales, ont installé le dieu, ont acheté des rizières, ont acquis des serviteurs ${ }^{38}$, ont créé des plantations, jlan ${ }^{39} \mathrm{ka}^{\prime} \mathrm{ol}^{40}$, avec leur effort humain ${ }^{41}$, ainsi que [celui] de [leurs] des-

présence d'un élément commun aux noms des deux Mratāñ (vinīta ${ }^{\circ}$ s'accorde bien avec l'hypothèse de deux demi-frères utérins - même s'il s'agit de noms d'initiation (voir n. 84).

${ }^{37} \mathrm{La}$ leçon $p \bar{\imath}$ est une variante de $p i$ (Long SeAm, p. 401). On la retrouve dans K. 158, A 1.1 et K. 158, D 1.9 (cette inscription [IC II, 99 et suiv.] montre aussi pi en même contexte, p. ex. face B, 1. 14); K. 175, S 1. 3; K. 198, b 1. 8; K. 393, N 1. 7. La distinction graphique $i / \bar{l}$ ne correspondait pas à une opposition phonologique en vieux khmer, ce qui suggère «that the vowel-length which was heard in these syllables and recorded in the writing was in fact a prosodic feature, occurring with zero final» (JACOB 1960, 354).

38 Le mot kñum (avec le sanskrit dāsa) a suscité une abondante littérature; voir, récemment, VICKERY 1998, 225 et suiv., et SANDERSON 2003-04, 395-400.

${ }^{39}$ Le sens de jlañ n'est pas connu. Le mot se rencontre juxtaposé à $k^{\prime} \mathrm{ol}(=\mathrm{ka}$ 'ol) également dans K. 79, 1. 9 et 12-13, où CEDÈs [IC II, 70 et suiv.] prend les deux mots ensemble comme toponyme. Il apparaît comme nom propre dans K. 149, 1. 5 [IC IV, 28]. Une nouvelle attestation de jlan figure dans Ka 79, 1.8 [NIC II-III, 198 et suiv.]: gi kṣetra ta nā jlañ a ásrama, que Pou traduit «les champs à Jlañ Āśrama». Pou 1992, 185 et suiv. présente sous la même entrée jalañ (K.155II, 1.13 [IC V, 66]) et la forme angkorienne jralon (K. 292C, 1. 14 [IC III, 211]), tous trois avec la même glose «voie, cours, torrent de montagne». Renonçant à ses interprétations de 1981 (p. 103) et 1982 (p. 177 et 192), Philip Jenner me signale dans une lettre du 17 décembre 2004 que: «Since 1981 I've

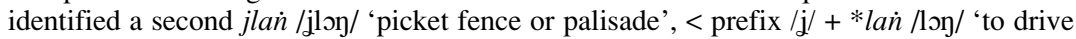
in, implant', which can be compared with modern janla'ń /con'lvəy/ 'stake'». Le présent contexte permettrait sans doute de considérer jlan comme un verbe selon l'interprétation de Philip Jenner, mais les autres attestations ne vont pas dans ce sens. Je ne vois pas de solution satisfaisante aux problèmes que pose ce mot, d'autant plus que le sens de ka'ol n'est pas certain non plus. Il est vraisemblable qu'il faille comprendre les mots jlan et $\mathrm{ka}$ 'ol ensemble.

${ }^{40} \mathrm{La}$ forme du mot assez répandu $\mathrm{ka}$ 'ol est fluctuante dans les inscriptions (on trouve aussi $k$ 'ol, $k$ 'ol, et même $k$ '”ol, voir Ka 11, 1. 12 [NIC II-III, 191-193 et pl. VI p. 318]), bien que les éditions n'indiquent pas toujours ce fait. En ce qui concerne la signification du mot, la glose «grenier» de LONG SEAM (p. 61) est proposée sans justification, mais elle est probablement inspirée par celle de JENNER 1982, 39 («granary»). Après d'autres tentatives (p. ex. Pou 1984, 107), Pou semble en 2001 (p. 193: «friche», avec point d'interrogation) être revenue à l'interprétation de CEDÈs, qui ne s'est exprimé qu'une seule fois sur le sens possible de ce mot: «[kaol] semble s'appliquer à une certaine espèce de terrain» $(I C \mathrm{~V}, 84, \mathrm{n} .1)$. À mon avis, cette dernière hypothèse reste toujours la plus vraisemblable, mais le terme nécessiterait une étude approfondie qui prenne en compte l'ensemble des occurrences.

${ }^{41}$ Le mot sanskrit purușakāra, assez répandu en Inde dans les textes épiques et les codes de dharma, signifie «actes, efforts de l'homme» par opposition au daiva, c.-à-d. le destin. Le mot fonctionne comme nom propre d'un vā dans K. 78, 1.18 [IC VI, 13]; K. 582, 1. 4-5 [IC II, 200]; K. 786, 1. 8 [IC VII, 107]. Par ailleurs, il apparaît encore dans le composé bhümipurușakāra de K. 249, 1. 8-9 [IC III, 98 et suiv.] que CEEDÈs traduit par 
cendants qui donnent, à savoir le Mratāñ Cāruvidya, avec le Mratāñ Sucaritānanda, [et] avec le Mratāñ Viditānanda, enfants du Mratāñ Vinītagaṇa, neveux utérins ${ }^{42}$ du Mratāñ Vinītavin, neveux utérins de la Tāñ Sthiradevi. Voici ce qu'ils donnent avec [leur] effort humain, ainsi que [celui] de [leurs] descendants, au (? $)^{43}$ dieu.

(12-14) La rizière au bord ${ }^{44}$ du bassin du dieu qu'[ils] ont acquise du Poñ 'Íśānapavitra ${ }^{45}$ [avec] une mesure d'or ${ }^{46}$; témoins le Mratāñ Bhap, le Mratāñ Rmmen, ainsi que l'assemblée au complet ${ }^{47}$.

«produits de la terre». (K. 254B, 1. 10 [IC III, 185] présente en khmer le terme ätmapurușakārya, «le produit (du travail) de mes gens» selon CEDÈs. Le passage où il figure oppose des biens obtenus par l'ätmapurușakārya à ceux obtenus par vrah karunāprasāda. Il est parallèle à la stance XIIab du sanskrit dans cette même inscription: yo rājakaruna $\bar{a}$ lavdhair vvasubhir vānyathārjitaih «au moyen des trésors reçus de la faveur du roi ou obtenus d'autre façon» (CEEDÈs). Il n'est pas exclu qu'il faille revoir l'interprétation du khmer à la lumière de ce parallélisme). Quoi qu'il en soit, dans le contexte de K. 249 purușakāra désigne sans doute les produits en tant qu'ils résultent du travail humain. Je dois à Philip Jenner (lettre mentionnée ci-dessus, n. 39) l'idée que l'expression puruṣakāra a ici une fonction adverbiale.

${ }^{42}$ L'expression (hapax) kanmoy sahodara semble être l'équivalent de l'expression kanmoy kaṃton «neveu utérin», sur laquelle voir VICKERY 1998, 262; 264 n. 17; 286 n. 88 «sister's son».

${ }^{43}$ La traduction de toute la portion de texte allant de la fin de la 1.8 jusqu'à dan் vrahha (1. 12) est entièrement hypothétique. De plus, elle présuppose d'émender dan vrahha, que je n'arrive pas à construire avec le contexte mais dont la lecture est certaine, en ta vrahha (cf. 1.20 pradāna ta vrah).

${ }^{44}$ Le mot jeǹ s'utilise pour dénoter le bord d'un cours d'eau ou d'une pièce d'eau (K. 811, 1. 2 [IC VI, 63] jen travań vrah; K. 18, 1. 5 [IC II, 146] sre jen cdin «rizière au bord de la rivière»); K. 1034D, 1. 8 [JACQUES 1970, 81] jen chdin khlen pau anle 1 «une parcelle sur le bord de la rivière Khlen Pau» (voir la note du traducteur).

${ }^{45} \mathrm{Ce}$ nom se retrouve, semble-t-il, seulement dans K. 79, 1.7 [IC II, 70], inscription qui contient aussi les seules autres attestations de la séquence jlan $\mathrm{ka}$ 'ol (voir n. 39). La datation de K. 79 (565 śaka, et non 561: voir VICKERY 1998, 430 et BILLARD, à paraître) ne permet pas d'identifier son Mratāñ 'İsānapavitra au Poñ de même nom de K. 1214 (648 śaka).

${ }^{46}$ Par «une mesure d'or» je rends le mot mās, pour lequel voir VICKERY 1998, 443.

${ }^{47}$ Le mot 'aval doit être comparé avec 'val que l'on trouve dans la séquence 'vai si sabhä 'val sin nau de K. 154, B 1. 12 [IC II, 124] (voir JАCOB 1960, 361 et suiv. sur la variation 'aval/'val, de type assez courant en vieux khmer). Malheureusement, cette phrase, représentée avec « $^{\mathrm{A}}$ vai Si Sabhā, ${ }^{\mathrm{A}}$ val Sin» par CEEDÈs, aide peu. Pou 1992, 3 connaît un mot 'val de l'angkorien, qu'elle décrit comme une marque emphatique du pluriel en citant K. 214, B 1. 10 [IC II, 204]; K. 344, 1. 42 [IC VI, 162]; K. 829, 1.10 [IC IV, 43]; K. 235, D 1. 17 [BEFEO 43, 89 = CEDÈs 1992, 200]. ChaKRAVARTI (1982, 8), citant la dernière attestation, et en plus K. 175N, 5 [IC VI, 176], explique: «in old Khmèr it signified the idea of totality, fullness». Ce mot a été discuté par CEEDÈs (et DUPONT), 


\section{(14-17) La rizière du/des Sevabhāra ${ }^{48}$ que le Poñ Śaṅkrakīrtti et le Poñ} Śaṅkragana ${ }^{49}$ ont cédée ${ }^{50}$ pour 2 mesures d'or — Le Kloñ Kandam, de leur famille ${ }^{51}$, a également ${ }^{52}$ cédé une rizière de trois $j e^{53}$; témoins le

BEFEO 15/2, p. 102 et 43, 146 = CEDÈs 1992, 257 n. 5, et a partout été traduit par «tout» dans les Inscriptions du Cambodge. C'est sans doute pour cela que LONG SEAM, p. 7, a proposé la glose hypothétique «les membres de l'assemblée au complet» pour la séquence de K. 154, que je suis également ici.

Vu le désaccord parmi les lexicographes en ce qui concerne le mot sin, d'autres traductions que «ainsi que» pour la séquence man sin peuvent être proposées, par exemple: «qui est ailleurs», «qui a déjà été mentionnée». On ne sait pas qui étaient les membres de l'assemblée en question, et ma traduction reste donc hypothétique.

${ }^{48}$ L'autre lecture possible, sebhagāra, de formation étrange, ne se trouve pas ailleurs. Il me semble donc préférable de choisir la leçon sevabhāra, terme qu'on trouve comme titre d'un «Pu Nen்» dans K. 137, 1. 1 [IC II, 116]. En ajoutant sa note 2, «Sevabhära est sans doute une simple épithète, signifiant "serviteur"», CEEDÈs [IC II, 117] pensait certainement à un composé sanskrit seva + bhära. Cette interprétation doit être correcte, bien que la connexion syntaxique du mot sevā «service» avec une forme (verbale ou nominale) de $b h r$ «porter» soit inconnue dans la littérature sanskrite disponible, sauf dans un passage du Rāmāyaṇa (2.58.38b), où seulement certains mss septentrionaux lisent gurusevābhrt (lecture rejetée dans l'édition critique de Baroda, t. 2, p. 352). La finale brève du premier membre de ce composé sevabhāra n'est pas sans parallèles en sanskrit même: voir RENOU 1961 § 76C, p. 86 (exemples: mäla-bhārin-, gañga-datta-). La nature exacte de la fonction de sevabhära reste obscure.

${ }^{49}$ Ces deux noms avec śañk(a) $\mathrm{ra}^{\circ}$ se retrouvent l'un dans K. 582, 1. 8 [IC II, 200], l'autre dans K. 154, A 1. 12 [IC II, 124]. L'inscription K. 582, qui relate l'installation d'un lingga appelé Kedāreśvara, avait été datée par CCEDÈs de l'an śaka $615=693$ de n. è.; en réalité elle date de 667 de n. è. (BILLARD, à paraitre). Cette nouvelle datation l'éloigne un peu plus de notre inscription, ainsi que de K. 154 (de 734 de n. è., voir ci-dessus, p. 14), où le dieu Kedāreśvara figure comme bénéficiaire du service du Mratāñ Devasvāmi. Pour résumer, la divinité pourrait être la même dans K. 582 et K. 154 (voir VICKERY 1998, 117), mais la distance chronologique entre K. 582 et notre inscription ne permet pas d'identifier les deux Śan̉k(a)rakītti. En revanche, le Mratãñ Devasvāmi de notre inscription (1. 20) et celui de K. 154 peuvent être la même personne. Enfin, notre Poñ Śañkragaṇa est peut-être identique au Poñ Śañkaragaṇa de K. 154.

${ }_{50}$ Le causatif pañjāhv a été interprété de façons diverses. Voir l'aperçu de VICKERY (1998, 290 et n. 98), dont je suis l'interprétation convaincante faite à propos de K. 726 [IC V, 75-80] (voir aussi Ka 40 et Ka 42 dans les NIC II-III, 204 et suiv., 209 et suiv.).

${ }^{51}$ On peut supposer que la famille en question est celle des Poñ Śankkrakīrtti et Śañkragana.

52 Sur 'ukka, voir JАСОВ 1991, 197 (où il faut cependant supprimer le mot sanskrit $u k r a$, inexistant, comme source du mot vieux khmer) et 223.

${ }^{53}$ Le terme technique je, qui indique une certaine mesure de production en riz (JENNER 1981, 97 et suiv.; 1982, 184) pourrait être glosé «panier» (voir Pou 1992, 190; LoNG SEAM, p. 241). 
Mratāñ Kulaśarmma, le Mratāñ Rmmen, ainsi que l'assemblée au complet.

(17-19) La rizière d'un $p \bar{a} d a^{54}$ et trois je que le Poñ Vinayavardhana a cédée; témoins le Poñ Kulabhūṣa, le Poñ Bhīmagaṇa [et] le Mratāñ Vivrta.

(19-21) La rizière que le Poñ Vinayavardhana, [avec] le Poñ Kantān, a donnée en échange ${ }^{55}$ au Mratãñ Devasvāmi ${ }^{56}$ — voilà ce que le Mratāñ a offert au dieu; témoins le Mratāñ Hariśarmma, officiant ${ }^{57}$, le Poñ Bhīmagana $^{58}$.

54 Sur l'utilisation du mot pāda voir JenNer 1981, 188; 1982, 330; Pou 1992, 310; LONG SEAM, p. 395.

${ }^{55}$ Le mot tor n'était pas encore attesté en khmer préangkorien, semble-t-il, avant la publication de Ka 79 par Pou dans NIC II-III (2001). Ni SAKAmoto ni Long SEAm ne l'ont enregistré; Pou 1992, 221 propose l'entrée tor, tvar, tur, mais sans exemple pour la première forme. Un cliché que m'a aimablement communiqué Bertrand Porte permet d'améliorer la lecture de l'inscription mentionnée. Je cite le mot tor en contexte (1. 3-5): ku niṣthura tnor kñum 'āśrama man. mratāñ sāmanta tor. kon. ku phoñ. 'is. ge 'ai ta 'áśrama. Malheureusement, même cette nouvelle lecture n'aide pas à comprendre la syntaxe de tor. Il semble que le correspondant angkorien tvar puisse être suivi directement du nom de ce contre quoi quelque chose est échangé - voir K. 221N, 1.24 [IC III, 58] et spécialement le cas de K. 222, 1. 7-8 [IC III, 61 et 63] tai th'yak ti ten tvan kantāl tvar kñum ta kaṃsten ti jvan ta vrah «Tai Thayak que Ten Tvan Kantāl échange contre un esclave du Kamsten pour l'offrir à la divinité» (CEDÈs). Si on adoptait la même construction ici («rizière que le P. V. a échangée contre le P. K. du M. D.»), on serait étonné de voir échangée une rizière contre un Poñ. L'interprétation adoptée ici, qui implique de suppléer la particule $n u$ « [avec]» après tor, m'a été proposée par Philip Jenner dans sa lettre déjà mentionnée. La syntaxe en est certes troublante, car la particule $n и$ que supplée Jenner semble plutôt précéder ce contre quoi quelque chose est échangé (voir K. 105, 1. 25 et 30 [IC VI, 184 et suiv.]), mais il semble quand même plus que probable que cette interprétation rend compte de l'intention de l'auteur du texte.

56 Voici encore un nom qui se retrouve dans K. 154 (cf. n. 49).

${ }^{57}$ Pour le mot pamjuh, voir K. 127, 1. 10 [IC II, 89]; K. 145, 1. 4 [IC VI, 72]; K. 18, 1. 25 [IC II, 147]; K. 814B, 1. 60 et suiv. [BEFEO XXXVII, 407 = CEEDÈs 1992, 109], et surtout K. 154, A 1.12 [IC II, 124]. Le sens «officiant», extrait de la glose de ce mot proposée par Long SEAm (p. 388), est vraisemblable - et le contexte de K. 814 (loñ yudhișthira purohita loñ nan paṃjuh loñ valadeva paṃjuh) pourrait indiquer que la fonction de pamjuh est proche de celle de purohita.

${ }^{58}$ II y a au moins deux autres traductions possibles: «témoin Hariśarmma, [qui est] l'officiant du Poñ Bhīmagaṇa»; «témoin Hariśarmma, officiant le Poñ Bhīmagaṇa» (cette dernière interprétation susciterait l'idée qu'au contraire des transactions dénotées par pañjāhv, celle qu'indique tor nécessiterait la présence d'un paṃjuh).

Journal Asiatique 293.1 (2005): 11-43 
(21-23) La/le Kloñ Tāñ ${ }^{59}$, le Poñ Śarapracanda, le Poñ Nandagup, eux à parts égales ont cédé un jardin à la Tāñ Sthiradevi; témoins le Mratāñ Vivrta, le Poñ Bhīmagaṇa, le Poñ Bhadragaṇa, le Poñ Nandasena.

(23-24) Voici le total: rizières [produisant] 15 tloñ $^{60}, 40$ cocotiers, 2 cents aréquiers ${ }^{61}$.

(24-31) Serviteurs: Ku Sammrddha et ses 2 enfants, Ku Priy et ses 3 enfants, Ku Kandak et ses 2 enfants, Ku 'Avai Rn̉nap et ses 2 enfants, $\mathrm{Ku}$ 'Ampā̄n et son enfant qui ont été acquis du Poñ Vidyāśîl — prix de la Ku: une valeur de 15 lin d'argent ${ }^{62}$; témoins Devāditya ${ }^{63}$ de Hańsapura $^{64}$, Das 'A ${ }^{65}$ de Vravuk ${ }^{66}$, le Mratāñ Rmmen, Samarāgrasin

59 Voir ViCKERY 1998, 215 (et 253) sur kloñ tāñ.

${ }^{60}$ Sur cette «mesure de capacité de paddy, de riz, et même de riz cuit, équivalent à 59 kg environ», voir la discussion de Pou 1984, 145 et suiv. (citant B. Ph. Groslier). Voir aussi VICKERY 1998, 305 et suiv., et 443.

${ }^{61}$ Le mot śada pourrait être une forme prakritique du sanskrit śata. Ce type de développement de consonne sourde en sonore n'a pas été noté explicitement par BHATTACHARYA 1991, 6 et suiv. pour l'épigraphie khmère, mais on trouve des exemples comme padigraha (pour pratigraha) et prīdi (pour prīti), p. 8 et 10. Voir aussi VON HINÜBER 2001, $\S 172-177,180$. Ou devrait-on l'attribuer à la prononciation sud-indienne? Quoi qu'il en soit, dans l'inscription préangkorienne K. 811 [IC VI, 63] figure la séquence - - - śada 1 ton் 20-1 (1. 4), où il est plus que probable qu'après l'énumération des rizières, il s'agit bien de «100 [aréquiers], 21 (ou 20?) cocotiers». La forme śata se trouve aussi dans des formules comparables, p. ex. K. 582 [IC II, 200] sre sanre kanlahha ton tnem 10 slā tnem śata I, «1/2 sanre de rizière, 10 cocotiers, 100 aréquiers» (CEDÈs).

${ }^{62}$ Sur la «mesure de poids» lin, voir Pou 1992, 419, qui considère le terme comme un emprunt au chinois (attestations préangkoriennes chez JENNER 1981, 264 et 1982, 457). Il semble bien que la transaction ne concerne que la Ku dernièrement nommée. Estce que 15 lin serait un prix attendu pour une Ku (avec enfant) ? Pourquoi trouve-t-on ici deux mots (tlai et 'argha), l'un khmer et l'autre emprunté au sanskrit, qui dénotent tous les deux le prix?

${ }^{63}$ Ce nom se rencontre aussi dans K. 162 ( $\mathrm{X}^{\mathrm{e}} \mathrm{s}$.), st. X [IC VI, 102], dans un passage où figurent en tout trois noms en ${ }^{\circ} \bar{a}$ ditya, discutés par VICKERY 1998, 183. Le personnage en question, qui aurait vécu dans les années śaka 710, pourrait donc difficilement être identifié au nôtre (śaka 648).

${ }^{64}$ Encore une fois, on rencontre un nom ne se trouvant ailleurs que dans l'inscription K. 154, où Haṅsapura (face A, 1. 10) est la résidence d'un Vrah, peut-être le dieu Kedāreśvara. Hańsapura pourrait donc être cherché dans les environs de Phum Komrieng (province de Kandal), où a été découverte K. 154, et de Trai Trak (province de Kompong Speu), où a été découverte K. 582 (voir n. 49 sur la connexion entre les deux).

${ }^{65}$ Il semble que les éléments das et 'añ forment un nom composé. À propos de l'inscription préangkorienne K. 357, CEDÈs (IC VI, 41) notait: «certains de ces gens [dont l'inscription donne la liste] (...) n'étaient pas désignés par les appellatifs $v \bar{a}$ ou $k u$, mais portaient des noms composés de vocables divers suivis du mot añ. Cette onomastique se 
'adah ${ }^{67}$ - Ku Nịsphala et ses 2 enfants; Ku Tamve et ses 4 enfants; $\mathrm{Ku}$ Kansec et son enfant; Ku Saṃ'ap et ses 3 enfants; Ku Thī et ses 2 enfants; 6 petits-enfants; Ku Panheṃ; Vā Mukhamātra; Vā Slac; Vā Caṃho; Vā N̦iṣthura; Vā Nandabhakti. Voici le total: 45 serviteurs en tout $^{68}$.

\section{Appendix I: The Date of the Inscription (by J. C. Eade)}

The planetary information to be extracted from the inscription may be tabulated as follows, where the planets named are assigned their conven-

retrouve dans d'autres inscriptions préangkoriennes [n. 2: Inscriptions du Cambodge, II pp. 52, 53, 116]». L'élément das est inconnu ailleurs en vieux khmer. Faut-il penser au sanskrit dāsa «esclave»?

${ }^{66}$ Il est possible que le nom Vravuk (hapax) soit une variante de Vravok, attesté à deux reprises dans l'inscription non datée K. 728 (VICKERY 1998, 101 et suiv., à la suite de CEEDÈs, la date du VIII ${ }^{\mathrm{e}}$ s.), 1. 2-3: dron் vrahh don் gi äçrama ai pañcarā gi miçrabhoga ta vrah kamratān añ vravok kaṃlun kudya ukk gi āyakta ta pamnos ta pjuh vrah kamratāin vravok «Le domaine du dieu, ainsi que les āçrama à Pañcarā, ont leurs biens réunis à ceux de V. K. A. Vravok. L'intérieur de l'enceinte relève de l'autorité des religieux ta pjuh de V. K. Vravok» (éd. et trad. de CEEDÈs, IC V, 83 et suiv.; mais voir aussi VICKERY 1998, 427). JACOB 1976, 31 (voir aussi tableau p. 35), se fondant sur les mots samruk «repoussé», sruk «inhabited area» et saṃlok «cooking» qui correspondent respectivement au khmer moderne /somrok/, /srok/, /somloz-somlok/, tous avec /-ok/, est amenée à penser que les graphies $u / o$ devant $-k$ final en préangkorien pouvaient représenter le même phonème. La variation Vravuk/Vravok en serait un bon exemple au niveau synchronique (cf. aussi le cas sahutra/sahodara relevé ci-dessus n. 35). Sur le nom (toponyme ?) Vravok, voir VICKERY 1998, 143.

${ }^{67}$ Le mot 'adah reste mystérieux. JENNER 1981, 359 et 390 oppose les formes 'dahl'adah/'adahl'adās (gloses: «constituent of slave-name», «male slave-name», «female slave-name»; à propos de la dernière, il ajoute «not necessarily Skt. dāsa») à un 'dah «unidentified» que l'on trouve dans K. 154, A 1. 14. Pou 1992, 10 groupe toutes ces formes (sauf 'adās, K. 600, E 1. 6 [IC II, 22]) ensemble sous la glose «heurter, frapper, boucher» (avec référence au khmer moderne dah /teah/); LONG SEAM, p. 2 donne (sans argument) la glose «témoin» pour l'attestation dans K. 154, A 1. 14, mais cette glose rendrait notre passage pléonastique. Quelqu'envie qu'on ait de faire appel encore une fois à K. 154, le mot 'dah qui y figure est lui-même très problématique (cf. CEDÈs, IC II p. 125 n. 3); selon CeEdès et Long SEAm, il y introduirait une liste de titulaires. S'il est néanmoins lié à notre 'adah, les deux formes présentent la même variation ' $a C /$ ' $C$ que celle relevée ci-dessus n. 47 ('aval/'val).

${ }^{68}$ Nous avons vu ci-dessus (n. 28) qu'il n'est pas possible de restituer deux noms à la transition des lignes 29 à 30 . Dans ces conditions, il semble également impossible de faire concorder le total ici noté avec celui qu'on obtient en additionnant les serviteurs et leur progéniture: 44 (11 Ku, $5 \mathrm{Vā}, 22$ enfants, 6 petits-enfants).

Journal Asiatique 293.1 (2005): 11-43 
tional number and listed against the signs of the zodiac assigned to them (indicated by clock position, for reading in the diagram): ${ }^{69}$

$\begin{array}{ll}\text { Lagna (L) } & \text { Scorpio (5 o'clock position) } \\ \text { Moon (2) } & \text { Sagittarius (4 o'clock) } \\ \text { Jupiter (5) } & \text { Sagittarius (do.) } \\ \text { Sun (1) } & \text { Capricorn (3 o'clock) } \\ \text { Mercury (4) } & \text { Capricorn (do.) } \\ \text { Saturn (7) } & \text { Capricorn (do.) } \\ \text { Mars (3) } & \text { Aries (12 o'clock) } \\ \text { Venus (6) } & \text { Aquarius (2 o'clock) }\end{array}$

These positions can be tested by computer program..$^{70}$ The choice of year is here determined by how the chronogram is read and in the event the resulting match demonstrates that the intended year is saka $648 .{ }^{71}$ The diagram places the planets in the signs ( $r a \bar{s} i)$ they occupied on Wednesday 25 December 726, the date that answers to 28 Paușa in śaka $648 .^{72}$ The diagram and the text are in entire agreement.

The positions tabulated - e.g. Sun 9 [signs] 5 [degrees] 19 [arcminutes] — are valid for midnight at Ujjain, the standard reference point. ${ }^{73}$

${ }^{69}$ For more information about the analysis and system of representation, cf. EADE 1995, 78-82.

70 The program employed ("HIC": <chriseade@actewangl.net.au>) uses the 'old' Süryasiddhānta (not the 'modern' one edited by Burgess). For its parameters, see BILlaRD 1971, 75 (and scattered entries in his Index). The program is Macintosh specific; the "Pancanga" program for PC made available by Michio Yano at <www.kyotosu.ac.jp/ yanom/pancanga $>$ generates similar results.

71 The word kośa has in other contexts been considered ambiguous, but it here stands in the hundreds position, not the tens or units, and 548 is not in the reckoning. Only the sun, Mercury and the moon would then be in the positions assigned to them.

72 The text is unusual in counting the day of the month past 15: the usual form would be krșna 13. [Gerdi Gerschheimer ajoute d'autres cas de ce type: K. 50 (de 667 de n. è.), K. 60 (626), K. 604 (627); voir aussi K. 55 st. XV (postérieure à 628); voir encore K. 260S.1 (921), K. 254 (1129) st. XIII. Ces quatre occurrences préangkoriennes vérifiables confirment que le mois commençait avec la quinzaine claire (schéma dit śuklādi ou amānta), ce qui semble du reste la norme avec l'ère śaka (cf. Kielhorn dans The Indian Antiquary 25 (Oct. 1896), p. 271-272).] It must be stressed to those unfamiliar with the procedures that the computer diagram is generated solely by giving the program the year-month-day of the text: the attendant planetary positions are found entirely independently of the original.

73 The ancient South East Asian equivalent of Greenwich was Ujjain, which served the same purpose of providing the zero in longitude for astronomical calculation. The 


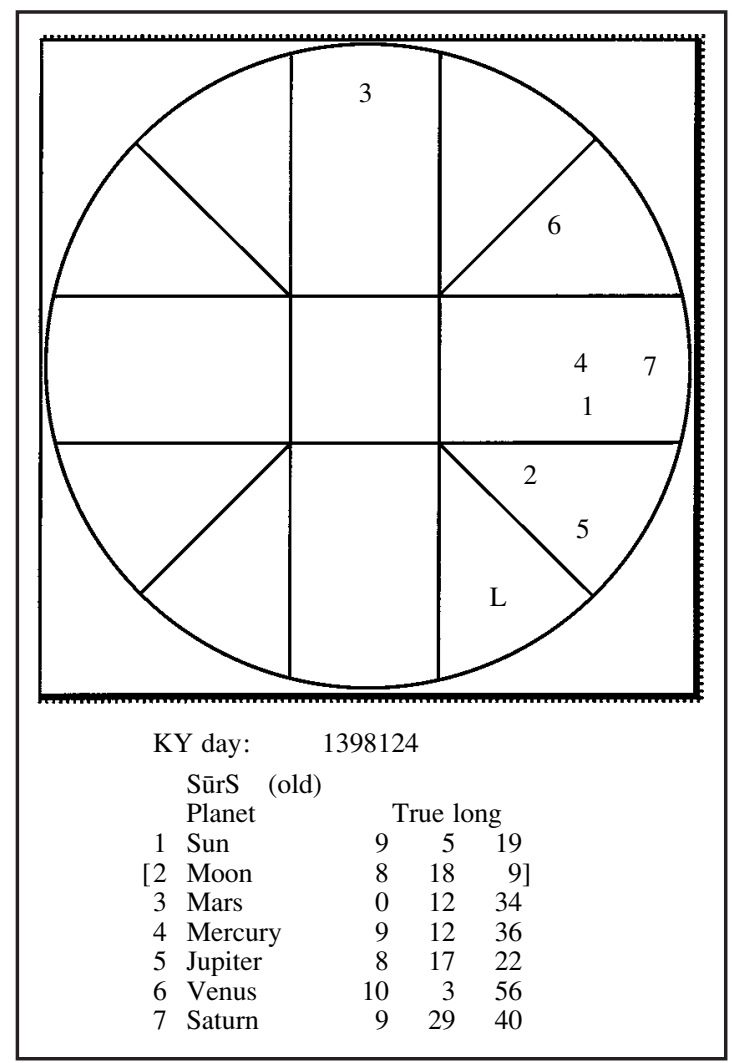

zero in latitude was supplied by a fictional island, Lanka, situated on the equator in the same longitude as Ujjain. Other locations were accommodated by establishing fixed adjustments to the Ujjain values. The problem is what adjustment to make for Cambodia. The lack of fine detail in the canon makes it necessary to use some appropriate form of rounding, and the value adopted here for 'Angkor' is 5 ghatikās $\left(=2\right.$ hours, $30^{\circ}$ East of Ujjain). Previous analyses have used the actual modern co-ordinates of Angkor proper, which introduces a false precision and a serious anachronism into the reckoning. Similarly some conversions of lagna to time have used a calculation scheme that takes account of the latitude of Angkor proper, again probably with a false precision and certainly with no means to verify its validity. It is more prudent, and generates no inadequacies in meeting the data, to use rounded values for both forms of reckoning. Those employed here for the lagna are still in use, and in Thailand astrological volvelles can be bought from which the relevant values can be read off directly once the volvelle has been set. 
And they do not in general (except for the moon) require to be adjusted for 'Angkor'. When times and positions do require adjustment the times are increased by 5 ghațikās ( 2 hours), as providing a realistic value that does not itself introduce a false precision. The Cambodians did not know for another 1000 years or so that Angkor was $101^{\circ} 40^{\prime}$ East of Paris (BARTH 1893, 589).

One notes that Saturn's tabulated position - 9s $29 \mathrm{~d} 40 \mathrm{~m}$ - is only 20 arcmins away from being in Aquarius not Capricorn, so it would have been no surprise to find the planet located by the inscription one rāsi in advance of the one actually given.$^{74}$ Strictly speaking, it could be argued, since positions are given only to the nearest $r \bar{r} s$ si, that the original reckoning could have placed Saturn way off line and (say) only at the very beginning of Capricorn. But an examination of the planetary positions given by the twenty horoscopes in the corpus points away from this sceptical view. The positions assigned to the outer planets (Mars, Jupiter, and Saturn) in the surviving horoscopes prove, with a tolerance of a couple of degrees, without exception either to be verified by computer or else to be explicable by means that do not involve the original calculation.

The placement of Scorpio as lagna allows a further refinement. The importance in the choice of lagna (the degree of the ecliptic on the eastern horizon, the ascendant) lay in its auspiciousness rather than the time it defined; though in this latter capacity it can be very useful for verification purposes. The interval between sunrise and the time chosen took account of the varying periods occupied by each räsis, on a scheme whose symmetry is plain when the times are expressed in ghațikās: ${ }^{75}$

74 Yano's value (n. 70 above) for Saturn is $10 \mathrm{~s}$ 0d $49 \mathrm{~m}$.

75 The relation of ghatikās to hours, minutes, seconds is the simple one of 60 to 24 . And since their day runs from $0 \mathrm{gh}$ to $60 \mathrm{gh}$ ( 6 a.m. to 6 a.m.), ghatikās are no more tedious to use than is hms. Their Wednesday $45 \mathrm{gh} 30$ would be our Thursday 00:12hrs, where using modern notation changes the name of the weekday.

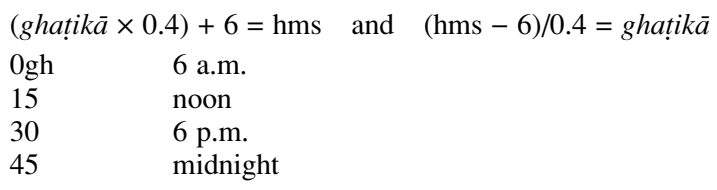




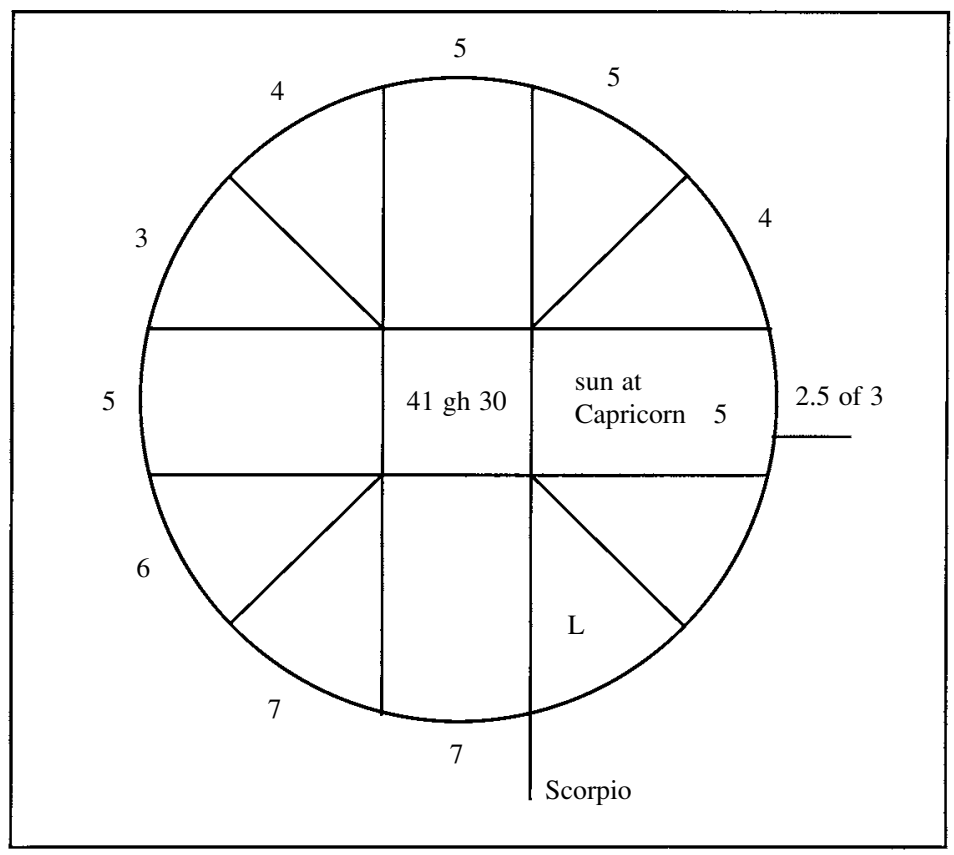

The only detail needed to convert lagna to auspicious time is the position of the sun in its rāssi. This then determines what proportion of the rāśi remained below the horizon at sunrise (fixed at 6 a.m.). In the present case the sun is 5 degrees into Capricorn, so 25 degrees have still to rise. As is always the case with sums of this kind, the Rule of Three applies:

$$
30^{\circ}: 5 \mathrm{gh}:: 25^{\circ}: n=2.5 \mathrm{gh}
$$

The total interval is $41 \mathrm{gh} 30$ (10:36 p.m.).

This value answering to the lagna's stated position can now be plotted against the times representing the moon's nakșatra and the tithi. The text places the moon (and Jupiter by association) in the first third of Sagittarius, where other similar expressions make it probable that the one-third of a rāśi was merely an equivalent, on the sun's circle (the ecliptic), for the moon's location on its circle (the nakșatras). The start 
of Sagittarius and the start of nakșatra 19: mūla coincide at $240^{\circ}$. Here the moon's rapid motion means that its Ujjain value must be adjusted by $5 \mathrm{gh}$ to bring it to 'Angkor':

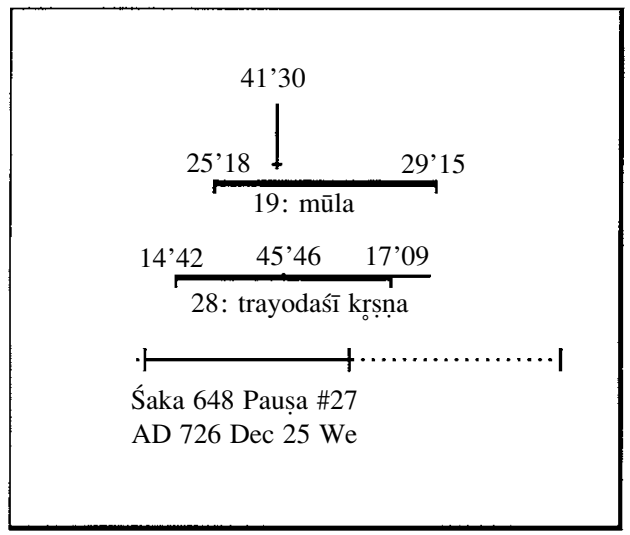

The moon is found to have entered müla at $25 \mathrm{gh} 18$, at which time the lagna was still only in Cancer (9 o'clock):

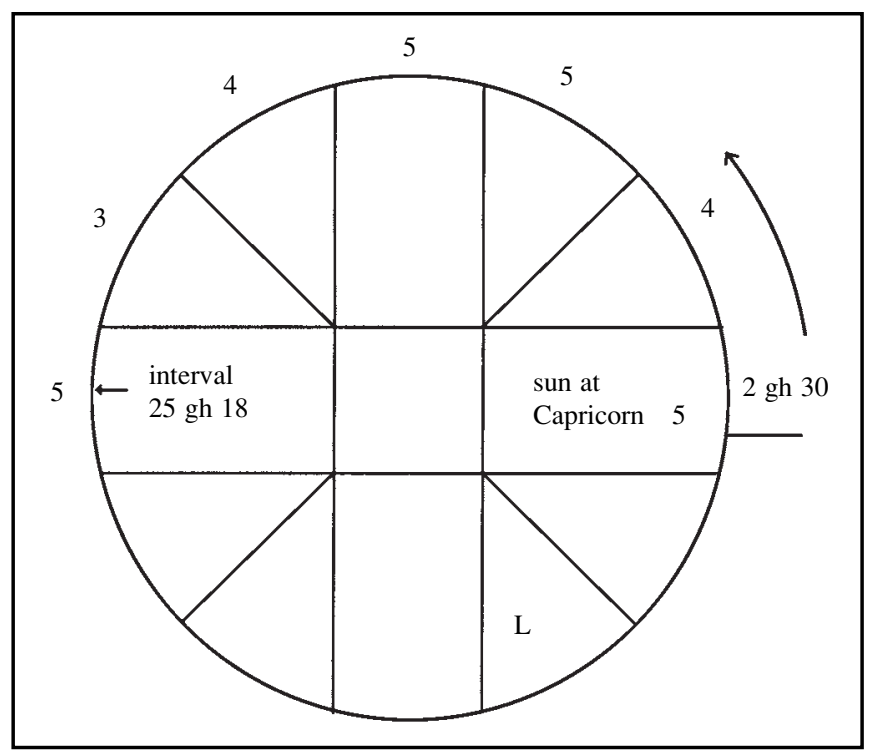

Journal Asiatique 293.1 (2005): 11-43 
For reasons that the inscription of course passes over, the rāsi $i$ Cancer to Libra were not selected. ${ }^{76}$

It is usual for the one-third rāśi position to be assigned only to the moon, and its being applied here to Jupiter, which was in fact in the middle of the rāsi $(8 \mathrm{~s} 17)$, not in the first third, is more likely to have been a poetic convenience than an error in calculating its position. ${ }^{77}$

It may be emphasised here that a replication of the original is possible only when the same procedures and something close to the same parameters are used by the computer program. Also that even with evidence that locates the planets no more precisely than to a given $r a \bar{s} i$, if the evidence is complete, as here, there is no possibility of mistaking one date for another. Had Saturn, for instance, here been located in Aquarius not Capricorn, it would not have led to a different date, because of the constraints supplied by the other planets.

$$
* * *
$$

Regarding K. 154, CEEDÈs (IC II, 124 n. 70) revised his view that the inscription dated to śaka 656 in favour of 606. The astronomical data, however, do not support the change. The constraints in addition to the year-month-day are the common ones of a weekday (Monday) and a nakșatra (uttarabhadra). The year śaka 606 had two Jyeșțhas, yielding Monday but the wrong nakșatra in nija Jyeștha; and uttarabhadra but the wrong weekday in adhika Jyeștha:

76 There were far more, and far more complex, considerations in the selection of an auspicious time than appear in the texts, as is also the case in Burma, Thailand, and Laos. Even such elementary factors as the particular propitiousness of a given nakșatra or lagna are not mentioned. They were good by their very choosing. There is, however, a preference noticeable for Taurus among the lagnas and for hasta and pusya among the naksatras.

77 The unit of a one-third rāssi has a significance astrologically as a 'decan' (drkāna), itself only one of the 16 divisions of a rāsi put to use by the astrologers (Brhatpārāsara, ch. 6, transl. Santhanam 1984-88). But these decans belong to the sun's circle, not the moon's, and the term was available to the Cambodian astrologers if they wanted to apply this technical sense. It seems therefore more likely that as applied to the moon the onethird rāssi is doing duty for the answering nakșatra, despite the fact that the two units are not directly commensurable, firstly because a naksatra covers $13^{\circ} 20^{\prime}$ not $10^{\circ}$ and secondly because many nakșatras cross rāśi boundaries. This latter ambiguity, however, would be cancelled by the time of day supplied by the lagna. 


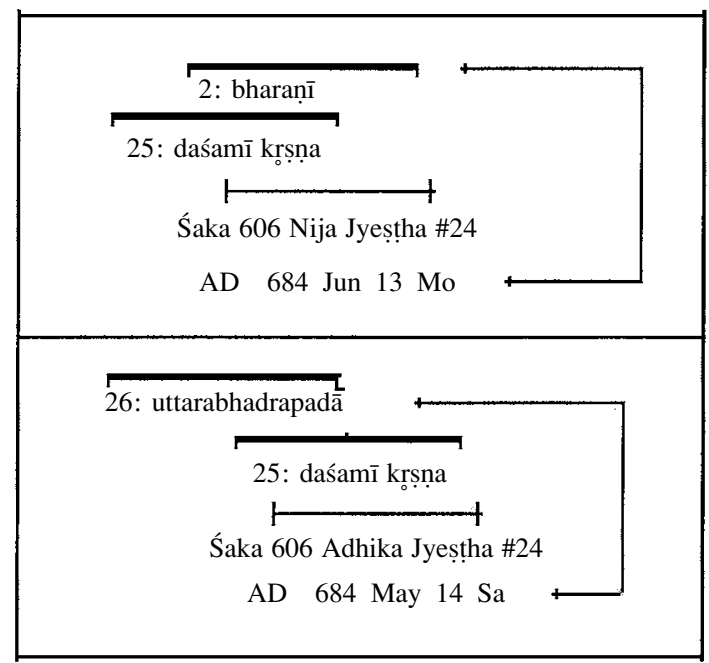

By contrast śaka 656 satisfies both constraints when Ujjain time is accelerated by $5 \mathrm{gh}$ to bring it to 'Angkor':

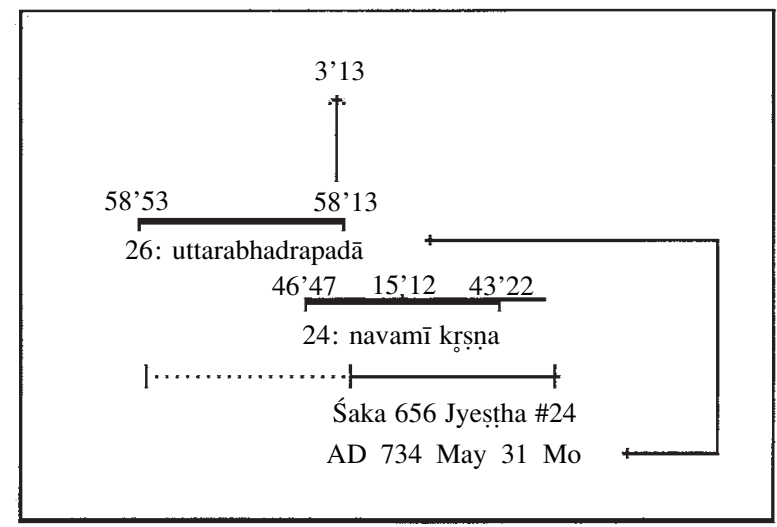




\section{Appendice II: Index onomastique}

Je rassemble ici les noms propres (anthroponymes, théonymes, toponymes) figurant dans l'inscription, avec éventuellement quelques commentaires. J'ai essayé de suivre dans leurs grandes lignes les exemples des Index de CEeDÈs (IC VIII) et de JACQUES (1971). Le signe $\diamond$ indique que je n'ai pas trouvé le nom en question dans ces deux Index ou dans celui de SAKAмото. Mais les deux premiers ne relèvent pas les noms de serviteurs, et le dernier ne concerne que les textes khmers publiés dans les $I C$ : il se peut donc que certaines attestations m'aient échappé. Les noms sont donnés dans l'ordre de l'alphabet indien, ce qui entraîne que l'occlusive glottale se trouve sous la «voyelle» qui est utilisée pour l'écrire.

Acyuta (épith.), 1

'Añ (voir Das 'Añ)

'Ampān $(\mathrm{Ku}), 25 \diamond$

'Avai (voir 'Avai Rñnap)

'Avai Rn்nap (Ku), $25 \diamond$

'İsāanapavitra (P.), 13

Kantān (P.), 19

Kandam $^{78}$ (Kloñ), 16

$\operatorname{Kandak}(\mathrm{Ku}), 25 \diamond$

Kansec (Ku), $28 \diamond$

\author{
Kulabhūṣa (P.), $18 \diamond$ \\ Kulaśarmma (M.), $16 \diamond$ \\ Cāruvidya (M.), $9 \diamond$ \\ Camho (Vā), 30 \\ Nișthura $^{79}$ (Vā), 30 \\ Nișphala (Ku), 28 \\ Tamve $^{80}(\mathrm{Ku}), 28$ \\ Tāñ ${ }^{81}$ (Kloñ?), 21 \\ Tribhuvaneśvara (d.), 5 \\ Thī (Ku), $29 \diamond$
}

${ }^{78}$ Ce nom apparaît aussi dans K. 137, 1. 18 [IC II, 116]; K. 562, 1. 12 [IC II, 197] et K. 689, B 1. 11 [IC VI, 48], où il appartient respectivement à un va et des $k u$.

${ }^{79}$ Sur ce type de «cérébralisation intempestive», qu'on rencontre aussi dans le nom Nisphala, voir les remarques sur «la confusion des dentales et des cérébrales» en tant que «trait de la prononciation moyen-indienne» de BHATTACHARYA 1991, 9 avec la note 47, et particulièrement la liste d'exemples dans la n. 58 (p. 11 et suiv.; tous exemples en contexte sanskrit); mais voir aussi sa remarque, p. 10: «Peut-être sommes-nous allé trop loin en voulant tout réduire au moyen-indien. Il faut tenir compte de la possibilité d'une évolution phonétique locale, parallèle à l'évolution moyen-indienne, mais non influencée par celle-ci».

${ }^{80}$ Ce nom apparaît p. ex. dans K. 18, 1.9 [IC II, 146] (ku tamve); K. 600, E 1.8 [IC II, 22] (ku tamve ru «Quasi active»?). Nulle part je n'ai trouvé kaṃve, autre lecture possible, cf. n. 27.

${ }^{81}$ Il n'est pas exclu que cet élément dans la séquence kloñ tāñ soit un nom propre. 
Das (voir Das 'Añ)

Das 'Añ (s. t.), $27 \diamond$

Devasvāmi (M.), 20

Devāditya (s. t.), 27

Nandagup $^{82}$ (P.), $22 \diamond$

Nandabhakti (Vā), $30 \diamond$

Nandasena (P.), $23 \diamond$

Panhem $^{83}(\mathrm{Ku}), 29 \diamond$

Priy $(\mathrm{Ku}), 25$

Bhadragana $^{84}$ (P.), $23 \diamond$

Bhap $^{85}$ (M.), 14

Bhīmagaṇa (P.), 18, 21, $23 \diamond$

Mukhamātra (Vā), 30

Rn்nap (voir 'Avai Rn்nap) $\diamond$

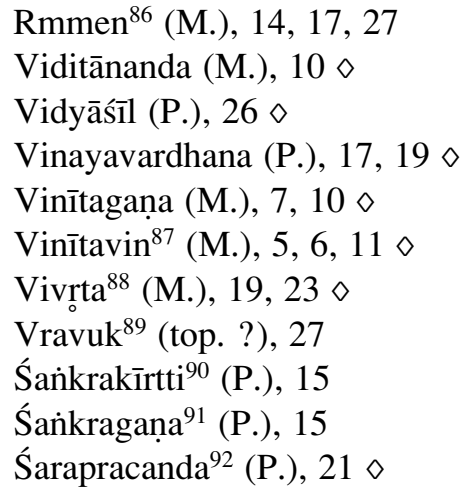

Sam’ap (Ku), 29

Sammrddha $^{93}(\mathrm{Ku}), 24 \diamond$

${ }^{82}$ L'élément ${ }^{\circ}$ gup (de skt. gupta) est bien connu dans l'épigraphie khmère; voir VICKERY 1998, 200 n. 87, qui donne plusieurs exemples.

${ }^{83} \mathrm{La}$ restitution de ce nom est soutenue par son occurrence assez fréquente dans le corpus angkorien; quant au préangkorien, voir K. 54, 1.10 [IC III, 159: va panhem], K. 451, S 1.7 et $\mathrm{N} 1.10$ [IC V, 50: ku 'me panhe I, vā panhem man col].

${ }^{84}$ Sur les noms en ${ }^{\circ}$ gana comme noms d'initiation çivaïtes, voir l'entrée de Goodall dans le Tāntrikäbhidhānakośa II (p. 174) et SANDERSON 2003-04, 398 n. 179. Il y a trois autres exemples dans cette inscription, à savoir les noms Bhīmagana, Vinītagaṇa et Śankragana.

85 Pour l'anthroponyme Bhap, voir vā bhap dans Ka 3 (préangkorienne, NIC II-III, p. 195), 1. 6; voir aussi gho bhap pi rmmel dans K. 134 (fin VIII ${ }^{\mathrm{e}}$ s.), 1. 18.

${ }^{86}$ Un Mratāñ Rmmen figure dans K. 424, A 1. 9 [IC II, 73]. Cf. aussi K. 155 II, 1.9 [IC V, 66].

${ }^{87}$ Ces deux derniers noms avec vinīta ${ }^{\circ}$ pourraient être comparés avec le nom d'un kñum vinìta attesté dans K. 1, 1. 12 [IC VI, 29]; K. 76, 1. 2 [IC V, 8]; et comme nom d'un Poñ dans K. 561, 1.18 [IC II, 42 (avec erreur soit dans le texte vinita, soit dans la traduction, p. 43 «Vinīta», forme sous laquelle le mot a été enregistré dans l'Index)].

${ }^{88}$ Cf. le nom d'une Ku Suvivrtā dans K. 555 I, 1. 12-13 [IC V, 66].

89 Voir la n. 66 ci-dessus.

${ }^{90}$ Ce nom se retrouve dans K. 582, 8 [IC II, 200].

${ }^{91}$ Ce nom se retrouve dans K. 154, A 1.12 [IC II, 124].

92 Sur l'orthographe avec dentales au lieu des rétroflexes attendues, cf. BHATTACHARYA 1991, 9 n. 47, où est cité, entre autres, un cas canda pour canda. Le nom («Formidable avec ses flèches») ne semble pas se retrouver dans l'épigraphie khmère. Je ne le connais pas non plus dans la littérature indienne, mais on peut le comparer avec pracandaśarakārmuka «ayant des flèches et un arc formidables», épithète du dieu de l'amour dans le Mahābhārata (passage 1730*, 1. 5 de l'édition critique, inséré dans quelques mss après 1.161.12).

${ }_{93}$ Ce nom peut être comparé avec le nom (de tai) Sa(ṃ)mrddhi qui est assez répandu 
Samarāgrasin $^{94}$ (s. t.), $27 \diamond$

Sucaritānanda (M.), $9 \diamond$

Sthiradevi (Tāñ), 6, 11, $22 \diamond$
Slac $(\mathrm{V} \overline{\mathrm{a}}), 30 \diamond$

Hańsapura (top.), 27

Hariśarmma (M.), 21

\section{Bibliographie}

AYMONIER, Étienne

1904 Le Cambodge, III. Paris: Ernest Leroux.

BARTH, Auguste

1885 Inscriptions sanscrites $d u$ Cambodge. Notices et extraits de la Bibliothèque nationale et autres bibliothèques, publiés par l'Institut national de France. Tome XXVII (1 ${ }^{\text {re }}$ partie). $1^{\text {er }}$ fascicule [Paris: Imprimerie nationale], 1-180.

1893 «Note additionnelle au sujet des dates contenues dans les inscriptions du Cambodge du $1^{\text {er }}$ fascicule et dans les inscriptions de Campā», Notices et extraits (...). Tome XXVII ( $1^{\text {re }}$ partie). $2^{\mathrm{e}}$ fascicule [Paris: Imprimerie nationale], 589-604.

Bergaigne, Abel

1882 «Les inscriptions sanscrites du Cambodge. Examen sommaire d'un envoi de M. Aymonier par MM. Barth, Bergaigne et Senart. Rapport à M. le Président de la Société asiatique», JA 20, 139-194.

BHATTACHARYA, Kamaleswar

1991 Recherches sur le vocabulaire des inscriptions sanskrites du Cambodge. Paris: EFEO (PEFEO 167).

BILLARD, Roger

1971 L'Astronomie indienne: investigation des textes sanskrits et des données numériques. Paris: EFEO (PEFEO 83).

à paraître «Dates des inscriptions du pays khmer», éd. par J. C. EADE.

ChaKravarti, Adhir

1982 «A Glossary of Old Khmer» (1 ${ }^{\text {re }}$ série, sans page de titre), PraciBhasha-Vijnan / Indian Journal of Linguistics 9.1, 1-16 (numérotés indépendamment) [Añve-Camnā̄r] et 9.2, 46-53 [Campan்-Catussneha].

(selon SAKамото: K. 157D, 1. 21; K. 343N, 1. 20; K. 706N, 1. 17; K. 809N, 1. 45). Même variation dans les toponymes samrddhapura (K. 467, 1. 25 [IC III, 219]), samrddhipura (K. 292C, 1. 49 [IC III, 213]), samprddhipura (K. 754B, 1. 28 [BEFEO 36, 5 = CEEDÈs 1989, 286]), sammrrddhigrāma (K. 420, 1. 14 [IC IV, 162]).

${ }_{94} \mathrm{Ce}$ nom, du sanskrit samarägrasiṃha «lion dans le front du combat», n'est pas luimême attesté dans le corpus des inscriptions khmères, mais on peut le comparer à Samarasin (K. 292D, 1. 26 [IC III, 214]). 
CEDÈs, George

1918 «Études cambodgiennes, XII-XVI», BEFEO 18.9, 1-28.

1937-66 Inscriptions du Cambodge I-VIII. Paris: EFEO (Collection de textes et documents sur l'Indochine III).

1989 Articles sur le pays khmer. Tome I. Paris: EFEO.

1992 Articles sur le pays khmer. Tome II. Paris: EFEO.

EADE, J. C.

1995 The Calendrical Systems of Mainland South-East Asia. Leiden: Brill. FERLuS, Michel

1992 «Essai de phonétique historique du khmer (Du milieu du premier millénaire de notre ère à l'époque actuelle)», Mon-Khmer Studies 21, 57-89.

Headley, Robert K. Jr., Kylin Chhor, Lam Kheng Lim, Lim HaK Kheang, CHEN CHun

1977 Cambodian-English Dictionary. 2 tomes. Washington, D.C.: The Catholic University of America Press. [Réimpr.: Modern Cambodian-English Dictionary, Springfield/VA: Dunwoody Press 1997].

HINÜBER, Oskar von

2001 Das ältere Mittelindisch im Überblick. 2., erweiterte Auflage. Wien: Verlag der Österreichischen Akademie der Wissenschaften.

IC = Inscriptions du Cambodge; voir CEDÈs 1937-66.

$I M A=$ Inscriptions modernes d'Angkor; voir LEWITZ 1974.

ISC = Inscriptions sanscrites du Cambodge; voir BARTH 1885.

JACOB, Judith M.

1960 «The Structure of the Word in Old Khmer», BSOAS 23.2, 351-368. [= 1993, 1-17]

1976 «An Examination of the Vowels and Final Consonants in Correspondences between Pre-Angkor and Modern Khmer», dans Nguyen Dang Liem (éd.), South-East Asian Linguistic Studies, Vol. 2 (Canberra: ANU [Pacific Linguistics, series C - No. 42]), 19-38. [= 1993, 87-102]

1991 «A Diachronic Survey of some Khmer Particles (7th to 17th centuries)», dans Jeremy H. C. S. Davidson (éd.), Austroasiatic Languages. Essays in honour of H. L. Shorto (London: SOAS, University of London), 193-225. [= 1993, 179-211]

1993 Cambodian Linguistics, Literature and History. Collected Articles. Edited by David A. Smyth. London: SOAS.

JACQUES, Claude

1970 «Études d'épigraphie cambodgienne. IV. Deux inscriptions du Phnom Bakhen (K. 464 et K. 558). V. La stèle du Prasat Cha Chuk (K. 1034)», BEFEO 57, 57-89.

1971 «Supplément au tome VIII des Inscriptions du Cambodge», BEFEO 58, 177-195. 
1986 Compte rendu de JENNER, A Chresthomathy of pre-Angkorean Khmer, tomes II et IV (University of Hawaii 1981-82), BEFEO 75, 315-318.

1999 «Les inscriptions du Phnom Kbal Spãn (K 1011, 1012, 1015 et 1016). Études d'épigraphie cambodgienne - XI», BEFEO 86, 357-374.

JENNER, Philip N.

1981 A Chresthomathy of Pre-Angkorian Khmer II. Lexicon of the Dated Inscriptions. University of Hawai at Manoa: Center for Southeast Asian Studies. School of Hawaiian, Asian, and Pacific Studies (Southeast Asia Paper No. 20, Part 2).

1982 A Chresthomathy of Pre-Angkorian Khmer IV. Lexicon of the Undated Inscriptions. University of Hawai at Manoa: Center for Southeast Asian Studies. School of Hawaiian, Asian, and Pacific Studies (Southeast Asia Paper No. 20, Part 4).

LEWITZ, Saveros

1974 «Inscriptions modernes d'Angkor 35, 36, 37 et 39», BEFEO 61, 301-337.

Lingapurāṇa

Śrī-Vyāsa-maharșiproktam Śrī-Lingamahāpurānam, with the Sanskrit commentary Sivatoṣiṇi by Gaṇeśa Nātu. [Éd. par] Gañgāviṣnu (son of Krṣnadāsa). Bombay: Venkatesvara Press, V.S. 1981 [= 1924 AD]. [Réimpr., avec une Ślokānukraman̄ī par Nāgaśaraṇa LONG SEAM

Simha, Delhi: Nag Publishers, 1989 (2éd. 1996).]

s.d. Dictionnaire du khmer ancien (D'après les inscriptions du Cambodge du VIe. - VIIIe siècles). Fondation TOYOTA du Japon, imprimé Mahābhārata par le Phnom Penh Printing House.

The Mahābhārata. For the first time critically edited by V. S. Sukthankar and others. 19 tomes. Poona: BORI, 1927-59.

NIC $=$ Nouvelles inscriptions du Cambodge; voir PoU 1989 et 2001.

PELliot, Paul

1951 Mémoires sur les coutumes du Cambodge de Tcheou Ta-Kouan. Paris: Librairie d'Amérique et d'Orient.

Pou, Saveros

1984 «Lexicographie vieux-khmere», Seksa Khmer 7, 67-175.

1989 Nouvelles inscriptions du Cambodge I. Traduites et éditées. Paris: EFEO.

1992 Dictionnaire vieux khmer-français-anglais. Paris: Cedoreck.

2001 Nouvelles inscriptions du Cambodge II \& III. Traduites et éditées. Paris: EFEO.

Rāmāyaṇa

The Vālmìki-Rāmāyaṇa. Critically edited for the first time by G. H. Bhatt and others. 7 tomes. Baroda: Oriental Institute, 1960-75. 
RENOU, Louis

1961 Grammaire sanscrite. Tomes I et II réunis. ... Deuxième édition revue, corrigée et augmentée. Paris: Adrien Maisonneuve.

SAKAмото, Yasuyuki

Kodai Kumērugo: KWIC sakuin (Old Khmer: KWIC index). Compiled privately for the use of the author.

SANDERSON, Alexis

2003-04 «The Śaiva Religion among the Khmers (part I)», BEFEO 90-91, 349-462.

SANTHANAM, R.

1984-88 Brihat Parasara Hora Sastra of Maharshi Parasara / Maharșiparāśarakrtabrhatpārāśarahorāśāstram. English translation, commentary, annotation, and editing. Tomes I \& II. New Delhi: Ranjan Publications.

SHORTO, H. L.

1971 A Dictionary of the Mon Inscriptions from the sixth to the sixteenth Centuries. London: Oxford University Press.

Tāntrikābhidhānakośa

Tāntrikābhidhānakośa II. Dictionnaire des termes techniques dans la littérature hindoue tantrique, sous la direction de H. Brunner, G. Oberhammer et A. Padoux. Wien: Verlag der Österreichischen

Vāmanapurāṇa Akademie der Wissenschaften, 2004.

The Vāmana Purāna. Critically edited by Anand Swarup Gupta. Varanasi: All-India Kashiraj Trust, 1967.

Vāyupurāṇa

The Vāyumahāpurānam. Éd. par Khemarāja. Delhi: Nag Publishers, 1983 [Réimpr. de l'édition Venkatesvara de 1895 AD].

VICKERY, Michael

1998 Society, Economics and Politics in Pre-Angkor Cambodia. The 7th8th Centuries. Tokyo: The Centre for East Asian Cultural Studies for Unesco, The Toyo Bunko.

Vong Sotheara (Van’'Sudhārāā)

2003 «Silācārịk duol trabāmin saṃrón tūc (ka. 3112) nau khăṇ tañko rājadhānī bhnam beñ» [Inscription de Tuol Trapeang Samrong Toch dans l'arrondissement de Dangkao, Phnom Penh], Dassanāvattī Sañgamasāstr-Manussasāstr, 33 ${ }^{\mathrm{e}}$ année, $\mathrm{n}^{\circ} 45$ (oct.-déc. YANG Baoyun 2003), 26-32.

1994 «Nouvelles études sur l'ouvrage de Zhou Daguan», dans F. Bizot (éd.), Recherches nouvelles sur le Cambodge. Paris: EFEO, p. 227234. 


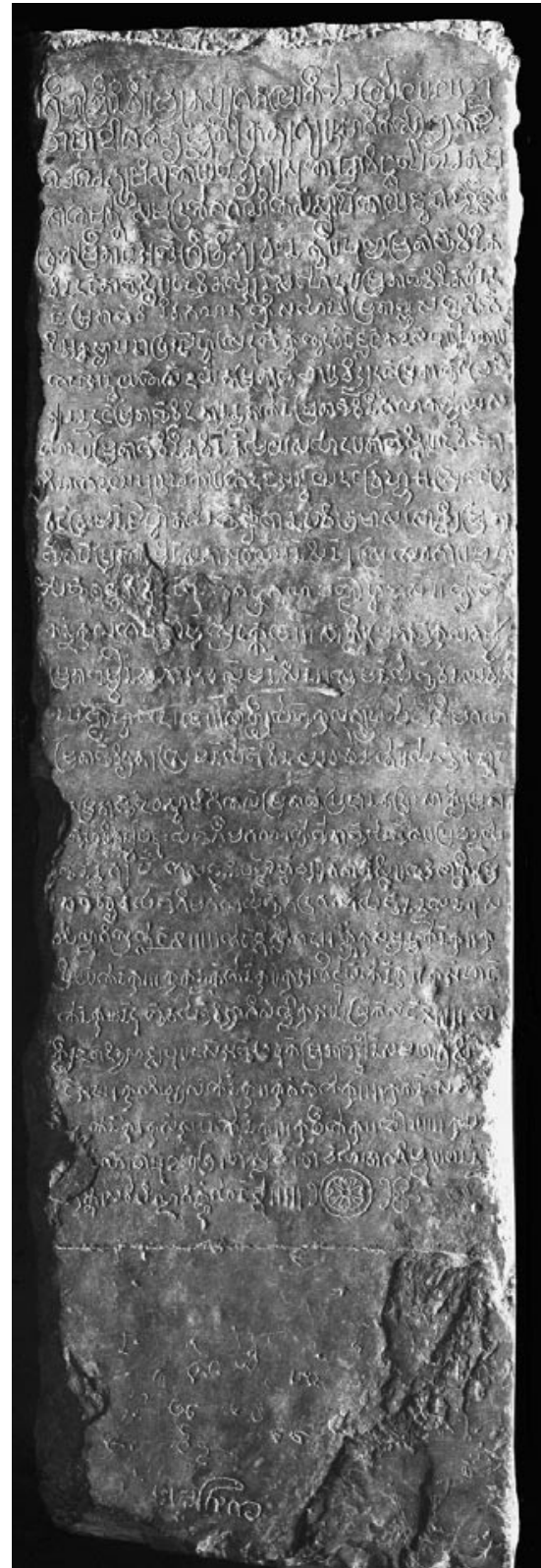

K. 1214

(cliché Atelier de restauration du MNPP / EFEO).

Journal Asiatique 293.1 (2005): 11-43 


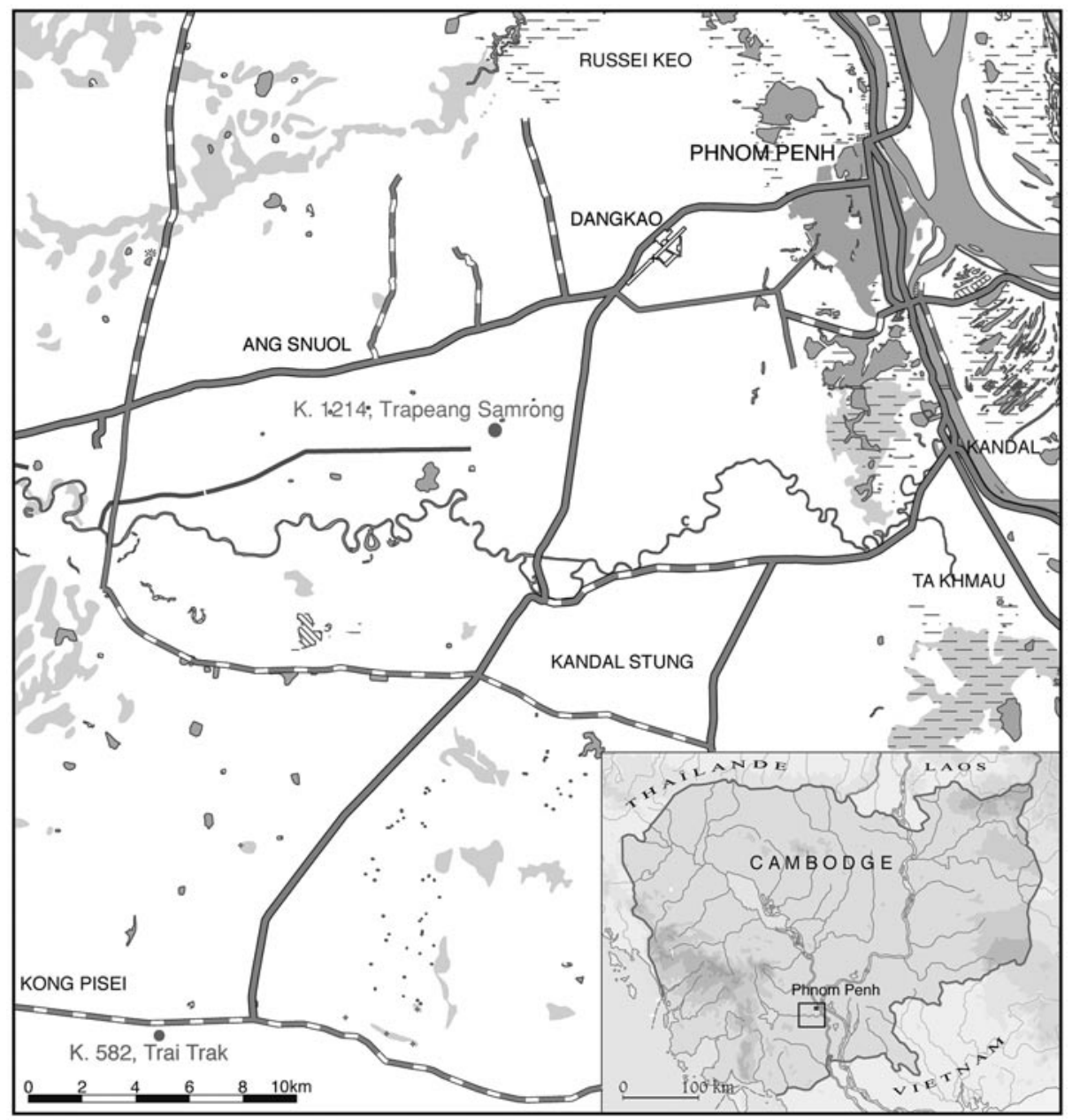

(EFEO / Ministère de la Culture et du Beaux-Arts du Cambodge). 